\title{
Rational Functions Invariant under a Finite Abelian Group
}

H.W. Lenstra, Jr. (Amsterdam)

\section{Introduction}

Let $k$ be a field and $A$ a permutation group on $n$ symbols $x_{1}, \ldots, x_{n}$. Then $A$ acts in a natural way as a group of $k$-automorphisms on the field of rational functions $k\left(x_{1}, \ldots, x_{n}\right)$. It is an old question, whether the field of invariants

$$
k\left(x_{1}, \ldots, x_{n}\right)^{A}=\left\{f \in k\left(x_{1}, \ldots, x_{n}\right) \mid g(f)=f \text { for all } g \in A\right\}
$$

is purely transcendental over $k$ or not, cf. Burnside, Theory of groups of finite order, second edition (1911), Ch. XVII. One usually calls this problem "Emmy Noether's conjecture" although Emmy Noether never stated that the answer would be affirmative [35-37].

Several positive results are known on this problem. Fischer [12] treated the case when $A$ is abelian and $k$ contains sufficiently many roots of unity. His result has been reproved $[25,7]$ and refined $[32,33]$ several times. If $\boldsymbol{A}$ is a $p$-group, where $p=\operatorname{char}(k) \neq 0$, then $k\left(x_{1}, \ldots, x_{n}\right)^{A}$ is purely transcendental over $k$, by $[21,20,22,16,33]$. Various groups of small order are treated in $[35,37,41,14,3-5,30,31,23,46,17]$.

Swan [44] and Voskresenskii [46] proved that $\mathbf{Q}\left(x_{1}, \ldots, x_{n}\right)^{A}$ is not purely transcendental over $Q$ if $A$ is a cyclic group of order $n=47$, permuting $x_{1}, \ldots, x_{n}$ transitively. An even smaller example is given by $n=8$, cf. (7.2). Further results for abelian $A$ were obtained by Endo and Miyata [10] and Voskresenskii [47, 48].

Our main theorem $[27,19]$ gives a complete solution for the case when $A$ is abelian and transitive. In this case we can index the $x_{i}$ by the elements of $A$ such that $g\left(x_{h}\right)=x_{g h}$ for all $g, h \in A$; we denote the field $k\left(\left\{x_{g} \mid g \in A\right\}\right)^{A}$ by $k_{A}$. Before stating the main theorem, we introduce some terminology.

Let $\rho$ be a finite cyclic group of order $m$ with generator $\tau$, and let $\Phi_{m} \in \mathbf{Z}[X]$ be the $m$-th cyclotomic polynomial. The ideal $\Phi_{m}(\tau) \mathbf{Z}[\rho] \subset \mathbf{Z}[\rho]$ $(=$ group ring of $\rho$ over $\mathbf{Z}$ ) does not depend on the choice of $\tau$, and we define

$$
\mathbf{Z}(\rho)=\mathbf{Z}[\rho] / \Phi_{\boldsymbol{m}}(\tau) \mathbf{Z}[\rho]
$$


Then $\mathbf{Z}(\rho) \cong \mathbf{Z}\left[\zeta_{m}\right]$, where $\zeta_{m}$ denotes a primitive $m$-th root of unity, so by [26, Ch. IV, Th. 4] the ring $\mathbf{Z}(\rho)$ is a Dedekind domain. The group of units of $\mathbf{Z}(\rho)$ contains $\rho$ in a natural way.

Denote by $k_{\text {cycl }}$ the maximal cyclotomic extension of the field $k$ inside an algebraic closure. Consider a subfield $K \subset k_{\text {cycl }}$ containing $k$ for which

$$
\rho_{K}=\operatorname{Gal}(K / k) \quad \text { is finite cyclic, with generator } \tau_{K},
$$

and let $p$ and $s$ satisfy

$$
p \text { is prime, } \quad 2 \neq p \neq \operatorname{char}(k), \quad s \in \mathbf{Z}, \text { and } s \geqq 1 .
$$

Then we define the $\mathbf{Z}\left(\rho_{K}\right)$-ideal $\mathfrak{a}_{K}\left(p^{s}\right)$ by

$$
\begin{array}{ll}
\mathfrak{a}_{K}\left(p^{s}\right)=\mathbf{Z}\left(\rho_{K}\right) & \text { if } K \neq k\left(\zeta_{p^{s}}\right), \\
\mathfrak{a}_{\boldsymbol{K}}\left(p^{s}\right)=\left(\tau_{K}-t, p\right) \subset \mathbf{Z}\left(\rho_{K}\right) & \text { if } K=k\left(\zeta_{p^{s}}\right), \text { where } t \in \mathbf{Z} \\
& \text { is such that } \tau_{\mathbf{K}}\left(\zeta_{p}\right)=\zeta_{p} .
\end{array}
$$

This definition does not depend on the choice of $\tau_{K}$.

For a finite abelian group $A$, put $m(A, p, s)=\operatorname{dim}_{\mathbf{Z} / p \mathbf{Z}}\left(p^{s-1} A / p^{s} A\right)$ (here $A$ is written additively), and

$$
\mathfrak{a}_{K}(A)=\prod_{p, s} \mathfrak{a}_{\mathbf{K}}\left(p^{s}\right)^{m(A, p, s)} \subset \mathbf{Z}\left(\rho_{K}\right),
$$

the ideal product ranging over all $p$ and $s$ satisfying (0.2).

Let $r(A)$ be the highest power of 2 dividing the exponent of $A$.

Main Theorem. Let $k$ be a field and let $A$ be a finite abelian group. Then the field

$$
k_{A}=k\left(\left\{x_{g} \mid g \in A\right\}\right)^{A}
$$

is purely transcendental over $k$ if and only if the following two conditions are satisfied:

(i) for every intermediate field $k \subset K \subset k_{\text {cycl }}$ for which (0.1) holds, the $\mathbf{Z}\left(\rho_{K}\right)$-ideal $\mathfrak{a}_{K}(A)$ is principal;

(ii) if $\operatorname{char}(k) \neq 2$, then $k\left(\zeta_{r(A)}\right)$ is a cyclic field extension of $k$.

Note that condition (ii) is satisfied if $\operatorname{char}(k) \neq 0$.

Sections 1-5 of the present paper are devoted to the proof of the main theorem. The idea is to use Fischer's result that $l_{A}$ is purely transcendental over $l$ if $l$ is a suitable cyclotomic extension of $k$. The "Galois descent" problem which arises in going from $l_{A}$ to $k_{A}$ is discussed, in a more general setting, in Sections 1 and 2 . Section 3 gives some useful technical information. The group $A$ does not occur in these sections. In Section 4 we show that we may assume that char $(k)$ does not divide 
the order of $A$, and Section 5 contains the proof of the main theorem. Supplementary results are given in Section 6, and some corollaries are indicated in Section 7.

The methods of this paper hardly exceed Galois theory and elementary commutative algebra. From cohomology of groups we need some facts on $H^{1}$ and $\hat{H}^{-\xi}$; these results are easily proved from the explicit descriptions of $H^{1}$ and $\hat{H}^{-1}$ given in [42, Ch. VII, VIII; 6, Ch. IV]. In the proof of (2.6) we need that a projective module over an abelian group ring has a rank; but this will be clear for the modules to which (2.6) is applied. We shall use freely the theory of finitely generated torsion free modules over a Dedekind ring [18]. Finally, the proofs of some corollaries in Section 7 require some algebraic number theory.

In the rest of this paper we write "rational" instead of "purely transcendental". A field extension $k \subset L$ is called "stably rational" if there exists a field extension $L \subset L^{\prime}$ of finite transcendence degree such that $L$ ' is rational over both $L$ and $k$. It is unknown whether "stably rational" implies "rational" $[40,9,34]$.

The notations $\Phi_{m}, \zeta_{m}, \mathbf{Z}(\rho)$ and $k_{A}$ have been introduced above. The characteristic of a field $k$ is denoted by $\operatorname{char}(k)$, the degree of a field extension $k \subset l$ by $[l: k]$ and the group of a Galois extension $k \subset l$ by $\mathrm{Gal}(l / k)$. For a prime $p$, a $p$-group is a group whose order is a power of $p$. The exponent of a group is the lowest common multiple of the orders of its elements. If a group $\pi$ acts on a set $S$, then $S^{\pi}=\{s \in S \mid \forall \sigma \in \pi: \sigma(s)=s\}$. The action of $\pi$ on $S$ is called trivial if $S=S^{\pi}$ and faithful if for every $\sigma \in \pi, \sigma \neq 1$, there is an $s \in S$ with $\sigma(s) \neq s$. By a $\pi$-module we mean a left module over the group ring $\mathbf{Z}[\pi]$, and we write $\otimes_{\pi}$ and Hom Hinstead of $\otimes_{\mathbf{z}[\pi]}$ and $\operatorname{Hom}_{\mathbf{z}[\pi]}$, respectively. The group of units of a ring $R$ with 1 is denoted by $R^{*}$. If $M$ is a module and $t$ is a nonnegative integer, then $M^{t}$ denotes the direct sum of $t$ copies of $M$; the only exception is the definition of $a_{K}(A)$ above, where we mean ideal power. Set theoretic difference is denoted by $\backslash$, and $|S|$ is the cardinality of a set $S$. The end or the absence of a proof is marked by $\mathrm{C}$.

\section{Permutation Modules and Rationality of Field Extensions}

Let $\pi$ be a finite group. A $\pi$-module is called a permutation module if it is free as an abelian group and has a Z-basis which is permuted by $\pi$. For example, free $\pi$-modules are permutation modules, and $\mathbf{Z}$, with trivial $\pi$-action, is a permutation module.

Every permutation module is a direct sum of modules $\mathbf{Z}\left[\pi / \pi^{\prime}\right]$; here $\pi^{\prime} \subset \pi$ is a subgroup and

$$
\mathbf{Z}\left[\pi / \pi^{\prime}\right]=\mathbf{Z}[\pi] \otimes_{\pi^{\prime}} \mathbf{Z} \quad(\text { as } \pi \text {-module) }
$$


where $\pi^{\prime}$ acts trivially on $\mathbf{Z}$. We call a $\pi$-module $N$ permutation-projective if $N \oplus N^{\prime}$ is a permutation module for some $\pi$-module $N^{\prime}$. One can take $N^{\prime}$ to be finitely generated if $N$ is, cf. the proof of (1.2).

(1.1) Proposition. Let $N$ be a permutation-projective $\pi$-module. Then $\hat{H}^{-1}(\rho, N)=H^{1}(\rho, N)=0$ for every subgroup $\rho \subset \pi$.

Proof. Since any permutation module over $\pi$ is a permutation module over every subgroup $\rho \subset \pi$, we may assume $\rho=\pi$. Also we may take $N=\mathbf{Z}\left[\pi / \pi^{\prime}\right]$, for a subgroup $\pi^{\prime} \subset \pi$. Then by Shapiro's lemma $[6, \mathrm{Ch}$. IV, Prop. 2] we have $H^{1}(\pi, N)=H^{1}\left(\pi^{\prime}, \mathbf{Z}\right)=0$, and the proof for $\hat{H}^{-1}$ is analogous.

(1.2) Proposition. Let $N$ be a $\pi$-module. The following statements about $N$ are equivalent:

(a) $N$ is permutation-projective;

(b) for every $\pi$-homomorphism $M_{1} \rightarrow M_{2}$ which induces surjective maps $M_{1}^{\rho} \rightarrow M_{2}^{\rho}$ for all subgroups $\rho \subset \pi$, the induced map

$$
\operatorname{Hom}_{\pi}\left(N, M_{1}\right) \rightarrow \operatorname{Hom}_{\pi}\left(N, M_{2}\right)
$$

is surjective;

(c) if $L$ is a $\pi$-module such that $H^{1}(\rho, L)=0$ for all $\rho \subset \pi$, then every exact sequence of $\pi$-modules

$$
0 \rightarrow L \rightarrow M \rightarrow N \rightarrow 0
$$

splits.

Proof. (a) $\Rightarrow$ (b). We may take $N=\mathbf{Z}[\pi / \rho]$ for some subgroup $\rho \subset \pi$. Then the functors $\operatorname{Hom}_{\pi}(N,-)$ and $(-)^{\rho}$ are equivalent, and (b) follows.

(b) $\Rightarrow$ (c). Let $0 \rightarrow L \rightarrow M \rightarrow N \rightarrow 0$ be a sequence as in (c). By the exact sequence of cohomology, the map $M^{\rho} \rightarrow N^{\rho}$ is surjective for every subgroup $\rho \subset \pi$. Applying (b) to $M_{1}=M$ and $M_{2}=N$ we find that the sequence splits.

(c) $\Rightarrow$ (a). One easily constructs a permutation module $M$ over $\pi$ and a $\pi$-homomorphism $M \rightarrow N$ such that $M^{\rho} \rightarrow N^{\rho}$ is surjective for every $\rho \subset \pi$. Let $L$ be the kernel of $M \rightarrow N$. The exact sequence of cohomology of

$$
0 \rightarrow L \rightarrow M \rightarrow N \rightarrow 0
$$

and (1.1) show that $H^{1}(\rho, L)=0$ for every $\rho \subset \pi$. By (c), the sequence splits, and (a) follows.

Note the analogy with the well known characterization of projective modules as direct summands of free modules.

Let $l$ be a field, $M$ a free abelian group of finite Z-rank $r$, and $l[M]$ the group ring of $M$ over $l$. If $M$ is written multiplicatively and $\left\{b_{1}, \ldots, b_{r}\right\}$ 
is a $\mathbf{Z}$-basis for $M$, then

$$
l[M]=l\left[b_{1}, \ldots, b_{r}, b_{1}^{-1}, \ldots, b_{r}^{-1}\right] .
$$

Thus we see that $l[M]$ is isomorphic to the ring of Laurent polynomials in $r$ variables over $l$. It follows that $l[M]$ is a unique factorization domain with group of units $l[M]^{*}=l^{*} \cdot M$. We denote the field of fractions of $l[M]$ by $l(M)$. This field is rational over $l$ of transcendence degree $r$.

Now suppose that $\pi$ acts faithfully on $l$ as a group of field automorphisms, and that $M$ has a $\pi$-module structure. We make $\pi$ act on $l[M]$ by

$$
\sigma\left(\sum_{m \in M} \lambda_{m} \cdot m\right)=\sum_{m \in M} \sigma\left(\lambda_{m}\right) \cdot \sigma(m), \quad \text { for } \sigma \in \pi,
$$

if $\lambda_{m} \in l$, and $\lambda_{m} \neq 0$ for only finitely many $m \in M$. The action is extended to $l(M)$ by $\sigma\left(a b^{-1}\right)=\sigma(a) \sigma(b)^{-1}$, for $a, b \in l[M]$, and $b \neq 0$.

In Theorem (1.7) we give a necessary and sufficient condition, in terms of $M$, that $l(M)^{\pi}$ be stably rational over $l^{\pi}$, cf. $[45,10]$. Theorem $(2.6)$ states that in a special situation this condition even implies that $l(M)^{\pi}$ is rational over $l^{\pi}$.

Remark that $l(M)^{\pi}$ is rational over $l^{\pi}$ if and only if a certain torus, defined over $l^{\pi}$ and splitting over $l$, is rational over $l^{\pi}$, cf. [38]. This will not be used in the sequel.

We usually write the group law in $M$ additively, although $M$ is a sub- $\pi$-module of the multiplicative group of $l(M)$.

(1.3) Proposition [43]. Let $W$ be an l-vector space on which $\pi$ acts semilinearly, i.e. $W$ is a $\pi$-module and $\sigma(\lambda w)=(\sigma \lambda) \cdot(\sigma w)$ for all $\sigma \in \pi, \lambda \in l$ and $w \in W$. Then $W^{\pi}$ contains an l-basis for $W$.

Proof. Put $S=\left(\sum_{\sigma \in \pi} \sigma\right) \in \mathbf{Z}[\pi]$. We show that $S W \subset W^{\pi}$ contains an $l-$ basis by proving that any $l$-linear function $\phi: W \rightarrow l$ annihilating $S W$ must be the zero function. Fix such a $\phi$, and fix $w \in W$. Then for every $\lambda \in l$ we have

$$
0=\phi(S \cdot \lambda w)=\sum_{\sigma \in \pi} \phi(\sigma w) \cdot \sigma(\lambda)
$$

By the linear independence of field automorphisms $[2, \mathrm{Ch} . \mathrm{V}, \S 7.5]$ we conclude $\phi(\sigma w)=0$ for all $\sigma \in \pi$. In particular $\phi(w)=0$, and (1.3) follows.

(1.4) Proposition [30]. Let $N$ be a finitely generated permutation module over $\pi$. Then $l(N)^{\pi}$ is rational over $l^{\pi}$.

Proof. Let $\left\{x_{1}, \ldots, x_{r}\right\} \subset l(N)^{*}$ be a $\mathbf{Z}$-basis for $N$ which is permuted by $\pi$. Applying (1.3) to $W=\left(\sum_{i=1}^{r} l \cdot x_{i}\right) \subset l(N)$ we find $y_{1}, \ldots, y_{r} \in l(N)^{\pi}$ such that $l\left(y_{1}, \ldots, y_{r}\right)=l(N)$. It follows that $l(N)^{\pi}=l^{\pi}\left(y_{1}, \ldots, y_{r}\right) . \quad[$ 
(1.5) Proposition. If $N$ is a permutation-projective $\pi$-module, and $0 \rightarrow M_{1} \rightarrow M_{2} \rightarrow N \rightarrow 0$ is an exact sequence of finitely generated $\mathbf{Z}$-free $\pi$-modules, then the fields $l\left(M_{2}\right)^{\pi}$ and $l\left(M_{1} \oplus N\right)^{\pi}$ are isomorphic over $l^{\pi}$.

Proof. The field $l\left(M_{1}\right)$ is naturally contained in $l\left(M_{2}\right)$. Let $l\left(M_{1}\right)^{*}$. $M_{2} \subset l\left(M_{2}\right)^{*}$ be the subgroup generated by $l\left(M_{1}\right)^{*}$ and $M_{2}$. Consider the exact sequence of $\pi$-modules

$$
0 \rightarrow l\left(M_{1}\right)^{*} \rightarrow l\left(M_{1}\right)^{*} \cdot M_{2} \rightarrow N \rightarrow 0
$$

where the map $f: l\left(M_{1}\right)^{*} \cdot M_{2} \rightarrow N$ is defined by

$$
f(\lambda \cdot m)=\left(m \bmod M_{1}\right) \in N, \quad \text { for } \lambda \in l\left(M_{1}\right)^{*} \text { and } m \in M_{2} .
$$

By Hilbert Theorem 90 and (1.2)(c) this exact sequence splits. The resulting $\pi$-homomorphism $N \rightarrow l\left(M_{2}\right)^{*}$ easily yields a field isomorphism $l\left(M_{1} \oplus N\right) \cong l\left(M_{2}\right)$ which respects the action of $\pi$, and (1.5) follows. Compare [39, Prop. 1.2.2].

(1.6) Proposition. If $N$ is a permutation module over $\pi$, and

$$
0 \rightarrow M_{1} \rightarrow M_{2} \rightarrow N \rightarrow 0
$$

is an exact sequence of finitely generated $\mathbf{Z}$-free $\pi$-modules, then $l\left(M_{2}\right)^{\pi}$ is rational over $l\left(M_{1}\right)^{\pi}$.

Proof. From (1.5) we get $l\left(M_{2}\right)^{\pi} \cong l\left(M_{1} \oplus N\right)^{\pi}$, and (1.4), applied to the base field $l\left(M_{1}\right)$ instead of $l$, says that $l\left(M_{1} \oplus N\right)^{\pi}$ is rational over $l\left(M_{1}\right)^{\pi}$.

(1.7) Theorem $[45,10]$. Let $M$ be a finitely generated $\mathbf{Z}$-free $\pi$-module. Then $l(M)^{\pi}$ is stably rational over $l^{\pi}$ if and only if there is an exact sequence of $\pi$-modules

$$
0 \rightarrow M \rightarrow N_{2} \rightarrow N_{1} \rightarrow 0
$$

in which $N_{1}$ and $N_{2}$ are finitely generated permutation modules.

Proof. If $0 \rightarrow M \rightarrow N_{2} \rightarrow N_{1} \rightarrow 0$ is an exact sequence as in the theorem, then $l\left(N_{2}\right)^{\pi}$ is rational over both $l^{\pi}$ and $l(M)^{\pi}$, by (1.6). This proves the "if"-part.

Next suppose $l(M)^{\pi}$ is stably rational over $l^{\pi}$, so

$$
l(M)^{\pi}\left(x_{1}, \ldots, x_{s}\right)=l^{\pi}\left(y_{1}, \ldots, y_{r+s}\right)
$$

where $\left\{x_{1}, \ldots, x_{s}\right\}$ is algebraically independent over $l(M)^{\pi}$ and $\left\{y_{1}, \ldots, y_{r+s}\right\}$ is algebraically independent over $l^{\pi}$. Let $\pi$ act on

$$
l(M)\left(x_{1}, \ldots, x_{s}\right)=l(M) \otimes_{l(M)^{\pi}} l(M)^{\pi}\left(x_{1}, \ldots, x_{s}\right)
$$

via the first factor. Put

$$
R_{1}=l[M]\left[x_{1}, \ldots, x_{s}\right] \text { and } R_{2}=l\left[y_{1}, \ldots, y_{r+s}\right]
$$


inside the field $l(M)\left(x_{1}, \ldots, x_{s}\right)$. By $[44$, Lemma 8] there are nonzero elements $a_{1} \in R_{1}^{\pi}$ and $a_{2} \in R_{2}^{\pi}$ such that $R_{1}\left[a_{1}^{-1}\right]=R_{2}\left[a_{2}^{-1}\right]$; call this last ring $R$. Lemma 7 of [44] tells us that there are exact sequences of $\pi$ modules

$$
\begin{aligned}
& 0 \rightarrow R_{1}^{*} \rightarrow R^{*} \rightarrow N_{1} \rightarrow 0 \\
& 0 \rightarrow R_{2}^{*} \rightarrow R^{*} \rightarrow N_{2} \rightarrow 0
\end{aligned}
$$

in which $N_{1}$ and $N_{2}$ are finitely generated permutation modules. Replacing $R^{*}, R_{1}^{*}$ and $R_{2}^{*}$ by $R^{*} / l^{*}, R_{1}^{*} / l^{*}$ and $R_{2}^{*} / l^{*}$ we get

$$
\begin{aligned}
& 0 \rightarrow M \rightarrow R^{*} / l^{*} \rightarrow N_{1} \rightarrow 0 \\
& 0 \rightarrow 0 \rightarrow R^{*} / l^{*} \rightarrow N_{2} \rightarrow 0 .
\end{aligned}
$$

The theorem follows.

(1.8) Corollary. Let $M$ be a finitely generated $\mathbf{Z}$-free $\pi$-module, and suppose $H^{1}(\rho, M)=0$ for every subgroup $\rho \subset \pi$. Then $l(M)^{\pi}$ is stably rational over $l^{\pi}$ if and only if $M \oplus N_{1} \cong N_{2}$ for certain finitely generated permutation modules $N_{1}$ and $N_{2}$.

Proof. (1.7) and (1.2)(c). $\quad \square$

\section{A Special Case}

In this section $\pi$ is a finite abelian group, and $l$ is a field on which $\pi$ acts faithfully as a group of field automorphisms. If $\pi^{\prime \prime} \subset \pi$ is a subgroup, then we call $\pi^{\prime}=\pi / \pi^{\prime \prime}$ a factor group of $\pi$. The canonical map $\pi \rightarrow \pi^{\prime}$ allows us to view every $\pi^{\prime}$-module as a $\pi$-module in a natural way.

Let $\rho$ be a cyclic factor group of $\pi$. Then there is a natural surjective ring homomorphism $\mathbf{Z}[\pi] \rightarrow \mathbf{Z}(\rho)$ (see the introduction for the definition of $\mathbf{Z}(\rho)$ ), which allows us to view every $\mathbf{Z}(\rho)$-module as a $\pi$-module. If $M$ is a $\pi$-module, we put

$$
F_{n, \rho}(M)=\left(M \otimes_{\pi} \mathbf{Z}(\rho)\right) /\{\text { elements of finite additive order }\} .
$$

Then $F_{n, \rho}$ is a functor from the category of $\pi$-modules to the category of torsion free $\mathbf{Z}(\rho)$-modules, left adjoint to an obvious functor the other way.

(2.1) Proposition. Let $S(\pi)$ denote the set of cyclic factor groups of $\pi$, and let $\pi^{\prime}$ be a factor group of $\pi$. Then there is a natural inclusion $S\left(\pi^{\prime}\right) \subset S(\pi)$, and for every $\pi^{\prime}$-module $M$ we have:

(i) if $\rho \in S\left(\pi^{\prime}\right)$, then $F_{\pi, \rho}(M) \cong F_{\pi^{\prime}, \rho}(M)$ over $\mathbf{Z}(\rho)$;

(ii) if $\rho \in S(\pi)$ but $\rho \notin S\left(\pi^{\prime}\right)$, then $F_{\pi, \rho}(M)=0$.

Proof. The inclusion $S\left(\pi^{\prime}\right) \subset S(\pi)$ is induced by the surjection $\pi \rightarrow \pi^{\prime}$. Assertion (i) is clear from $M \otimes_{\pi} Z\left[\pi^{\prime}\right] \cong M$. We prove (ii). Since $\rho \notin S\left(\pi^{\prime}\right)$, 
we can choose an element $\sigma \in \pi$, which has image 1 in $\pi^{\prime}$ while its image $\sigma^{*}$ in $\rho$ is $\neq 1$. Then $\sigma$ acts trivially on $M$, so

$$
\left(\sigma^{*}-1\right) \cdot\left(M \otimes_{\pi} \mathbf{Z}(\rho)\right)=0,
$$

where $\sigma^{*}-1$ is a nonzero element of $\mathbf{Z}(\rho)$. Since $\sigma^{*}-1$ divides some positive integer in $\mathbf{Z}(\rho)$, it follows that $M \otimes_{\pi} \mathbf{Z}(\rho)$ is torsion, so $F_{n, \rho}(M)=0$.

This proposition says that $F_{n, \rho}$ does not depend on $\pi$, in a certain sense. From now on we will write $F_{\rho}$ instead of $F_{n, \rho}$.

(2.2) Proposition. Let $N$ be a $\pi$-module, and $M \subset N$ a sub- $\pi$-module such that $N / M$ is a torsion group. Then $F_{\boldsymbol{\rho}}(M)$ is isomorphic to the image of $M$ under the natural map $N \rightarrow F_{\rho}(N)$, for every cyclic factor group $\rho$ of $\pi$.

Proof. Let $J$ be the kernel of $\mathbf{Z}[\pi] \rightarrow \mathbf{Z}(\rho)$. Then for every $\pi$-module $P$ there is a natural surjection $P \rightarrow F_{\rho}(P)$ with kernel

$$
\{p \in P \mid \exists k \in \mathbf{Z}, k \neq 0: k \cdot p \in J \cdot P\} .
$$

Since $N / M$ is torsion, we have

$\{m \in M \mid \exists k \in \mathbf{Z}, k \neq 0: k \cdot m \in J \cdot M\}=M \cap\{n \in N \mid \exists k \in \mathbf{Z}, k \neq 0: k \cdot n \in J \cdot N\}$, and (2.2) follows. $\quad$

(2.3) Proposition. If $N$ is a permutation module over $\pi$, then $F_{\rho}(N)$ is $\mathbf{Z}(\rho)$-free for every cyclic factor group $\rho$ of $\pi$.

Proof. It suffices to treat the case $N=\mathbf{Z}\left[\pi^{\prime}\right]$, where $\pi^{\prime}$ is a factor group of $\pi$. Then $F_{\rho}(N) \cong \mathbf{Z}(\rho)$ or $F_{\rho}(N)=0$, by $(2.1)$.

(2.4) Theorem. Let $\pi$ be a finite cyclic group, and $M$ a finitely generated projective $\pi$-module. Then the fields $l(M)^{\pi}$ and $l\left(\oplus_{\rho} F_{\rho}(M)\right)^{\pi}$ are isomorphic over $l^{\pi}$; here $\rho$ ranges over the set of cyclic factor groups of $\pi$.

The proof of this theorem is given at the end of this section. An analogous result is given in [10]. Compare also [11].

(2.5) Corollary. Let $\pi$ be a finite abelian group, and let $M$ be a finitely generated $\pi$-module of the form

$$
M=\bigoplus \pi_{\pi^{\prime}} M_{\pi^{\prime}}
$$

where each $M_{\pi^{\prime}}$ is a projective $\pi^{\prime}$-module, and where $\pi^{\prime}$ ranges over the set of cyclic factor groups of $\pi$. Then

$$
l(M)^{\pi} \cong l\left(\underset{\rho}{\oplus} F_{\rho}(M)\right)^{\pi} \quad \text { over } l^{\pi},
$$

with $\rho$ ranging over the set of cyclic factor groups of $\pi$. 
Proof. Let $\pi^{\prime}=\pi / \pi^{\prime \prime}$ be a cyclic factor group of $\pi$. Applying (2.4) to the cyclic group $\pi^{\prime}$, the module $M_{\pi^{\prime}}$ and the field $l^{\pi^{\prime \prime}}$, we find, using $(2.1)$ :

$$
l\left(M_{\pi^{\prime}}\right)^{\pi} \cong l\left(\underset{\rho}{\oplus} F_{\rho}\left(M_{\pi^{\prime}}\right)\right)^{\pi} \quad \text { over } l^{\pi}
$$

Tensoring with $/$ over $l^{\pi}$ gives an $l$-isomorphism

$$
l\left(M_{\pi^{\prime}}\right) \cong l\left(\oplus_{\rho} F_{\rho}\left(M_{\pi^{\prime}}\right)\right)
$$

which respects the action of $\pi$. Combination yields an $l$-isomorphism

$$
l(M) \cong l\left(\underset{\rho}{\oplus} F_{\rho}(M)\right)
$$

which respects the action of $\pi$, and (2.5) follows.

(2.6) Theorem. Let $\pi$ be a finite abelian group, and let $M$ be a finitely generated $\pi$-module of the form

$$
M=\underset{\pi^{\prime}}{\oplus} M_{\pi^{\prime}}
$$

where each $M_{\pi^{\prime}}$ is a projective $\pi^{\prime}$-module, and where $\pi^{\prime}$ ranges over the set of cyclic factor groups of $\pi$. Then the following three statements are equivalent:

(a) the field $l(M)^{\pi}$ is rational over $l^{\pi}$;

(b) the field $l(M)^{\pi}$ is stably rational over $l^{\pi}$;

(c) for every cyclic factor group $\rho$ of $\pi$, the $\mathbf{Z}(\rho)$-module $F_{\rho}(M)$ is free.

Proof. The implication (a) $\Rightarrow$ (b) is obvious.

(b) $\Rightarrow$ (c). Since $M$ is permutation-projective over $\pi$, we can apply (1.8). Using (2.3) we find that for every cyclic factor group $\rho$ of $\pi$ there exist finitely generated free $\mathrm{Z}(\rho)$-modules $P_{1}$ and $P_{2}$ such that $F_{\rho}(M) \oplus P_{1} \cong P_{2}$ over $\mathbf{Z}(\rho)$. Since $\mathbf{Z}(\rho)$ is a Dedekind domain, this implies that $F_{\rho}(M)$ is $\mathbf{Z}(\rho)$-free, as required.

(c) $\Rightarrow$ (a). Let $r\left(\pi^{\prime}\right)$ be the rank of $M_{\pi^{\prime}}$ over $\mathbf{Z}\left[\pi^{\prime}\right]$, and put

$$
N=\bigoplus_{\pi^{\prime}} \mathbf{Z}\left[\pi^{\prime}\right]^{r\left(\pi^{\prime}\right)}
$$

Let $\rho$ be a cyclic factor group of $\pi$. Then the $\mathbf{Z}(\rho)$-modules $F_{\rho}(M)$ and $F_{\rho}(N)$ are isomorphic; in fact, by assumption and by $(2.3)$, they are both $\mathbf{Z}(\rho)$-free of rank $\sum_{\pi^{\prime}} r\left(\pi^{\prime}\right)$, the sum ranging over those cyclic factor groups $\pi^{\prime}$ of $\pi$ for which $\rho$ is a factor group of $\pi^{\prime}$. Therefore

$$
\oplus_{\rho} F_{\rho}(M) \cong \underset{\rho}{\oplus} F_{\rho}(N)
$$


so $l(M)^{\pi} \cong l(N)^{\pi}$ by a twofold application of $(2.5)$. But $l(N)^{\pi}$ is rational over $l^{\pi}$, by (1.4), and (a) follows.

The remainder of this section is devoted to the proof of Theorem (2.4). We assume that $\pi$ is a cyclic group of order $m$ with generator $\tau$. The set of positive divisors of $m$ is denoted by $E(m)$. For $d \in E(m)$, the unique factor group of $\pi$ of order $d$ is denoted by $\pi_{d}$. If $C \subset E(m)$ is a subset, then we write $\Phi_{C}=\prod_{d \in C} \Phi_{d}$; for example, $\Phi_{E(m)}=X^{m}-1$. If $M$ is a $\pi$-module and $C \subset E(m)$, we write $M_{C}=M / \Phi_{C}(\tau) M$.

(2.7) Lemma. If $M$ is $\pi$-projective, and $d \in E(m)$, then $M_{E(d)}$ is permutationprojective over $\pi$.

Proof. The module $M_{E(d)} \cong M \otimes_{\pi} Z\left[\pi_{d}\right]$ is $\pi_{d}$-projective, hence a direct summand of $\mathbf{Z}\left[\pi_{d}\right]^{t}$ for some $t \in \mathbf{Z}, t \geqq 0$.

(2.8) Lemma. Let $M$ be $\pi$-projective, and $C, C^{\prime} \subset E(m)$ disjoint subsets. Then there is an exact sequence of $\pi$-modules

$$
0 \rightarrow M_{C} \rightarrow M_{C \cup C^{\prime}} \rightarrow M_{C^{\prime}} \rightarrow 0 .
$$

Proof. The map $M_{C \cup C^{\prime}} \rightarrow M_{C^{\prime}}$ is the natural one, and the map $M_{C} \rightarrow M_{C \cup \mathbf{C}^{\prime}}$ is induced by multiplication with $\Phi_{C^{\prime}}(\tau)$. For $M=\mathbf{Z}[\pi]$, exactness of the resulting sequence is easily checked. The general case follows since everything preserves direct sums.

Let $G(m)$ denote the set of all equivalence relations on $E(m)$. For $u \in G(m)$, we denote by $[u]$ the set of non-empty equivalence classes of $u$. Let $S(m) \subset G(m) \times G(m)$ be the set of $(u, v) \in G(m) \times G(m)$ for which

(2.9) there exist $d \in E(m)$ and $D \in[u]$, such that $E(d) \subset D, E(d) \neq D$ and $[v]=\{E(d), D \backslash E(d), C \mid C \in[u], C \neq D\}$.

(2.10) Lemma. The graph $(G(m), S(m))$ is connected.

Proof. The statement means that for all $u, v \in G(m)$ there is a finite sequence $\left(u_{j}\right)_{j=0}^{a}$ of elements of $G(m)$ such that $u_{0}=u$ and $u_{a}=v$, and such that for every $j$ with $0 \leqq j<a$, either $\left(u_{j}, u_{j+1}\right) \in S(m)$ or $\left(u_{j+1}, u_{j}\right) \in S(m)$. We call such a sequence a "path from $u$ to $v$ ".

Let the two "trivial" equivalence relations $i(m), w(m) \in G(m)$ be defined by

$$
[i(m)]=\{\{d\} \mid d \in E(m)\} \text { and }[w(m)]=\{E(m)\} \text {. }
$$

Clearly, it is sufficient to show that for each $u \in G(m)$ there is a path from $u$ to $i(m)$. This is done by induction on $m$. For fixed $m$, we use induction on $n(u)=|E(m)|-|[u]|$.

If $n(u)=0$ then $u=i(m)$ and obviously the required path exists. Suppose that $n(u)>0$, and let $e$ be the smallest element of $E(m)$ for which there exists a class $D \in[u]$ with $e \in D$ and $|D|>1$. Clearly $e<m$. Therefore, 
the induction hypothesis on $m$ may be applied, yielding a path $\left(v_{j}\right)_{j=0}^{b}$ from $i(e)$ to $w(e)$ in the graph $(G(e), S(e))$. For $0 \leqq j \leqq b$, let $D_{j} \in\left[v_{j}\right]$ be such that $e \in D_{j}$. Define $u_{j} \in G(m)$, for $0 \leqq j \leqq 2 b+1$, by

$$
\left[u_{j}\right]=\{C \in[u] \mid C \cap E(e)=\emptyset\} \cup\left\{D \cup D_{j}\right\} \cup\left(\left[v_{j}\right] \backslash\left\{D_{j}\right\}\right)
$$

if $0 \leqq j \leqq b$, and

$$
\left[u_{j}\right]=\{C \in[u] \mid C \cap E(e)=\emptyset\} \cup\{D \backslash\{e\}\} \cup\left[v_{2 b+1-j}\right]
$$

if $b+1 \leqq j \leqq 2 b+1$. We leave it to the reader to check that $\left(u_{j}\right)_{j=0}^{2 b+1}$ is a well defined path from $u=u_{0}$ to $u_{2 b+1}$, and that

$$
\left[u_{2 b+1}\right]=\{D \backslash\{e\},\{e\}\} \cup([u] \backslash\{D\}) .
$$

It follows that $n\left(u_{2 b+1}\right)=n(u)-1$, and the induction hypothesis on $n(u)$ yields a path from $u_{2 b+1}$ to $i(m)$. Combination yields a path from $u$ to $i(m)$. This proves the lemma.

Let $l$ and $M$ be as in (2.4). For $u \in G(m)$ we put $M(u)=\bigoplus_{C \in[u]} M_{C}$.

(2.11) Lemma. Let $u, v \in G(m)$. Then $l(M(u))^{\pi} \cong l(M(v))^{\pi}$ over $l^{\pi}$.

Proof. By Lemma (2.10) we may assume that (2.9) holds. Then by (2.8) there is an exact sequence of $\pi$-modules

Adding a summand

$$
0 \rightarrow M_{D \backslash E(d)} \rightarrow M_{D} \rightarrow M_{E(d)} \rightarrow 0 .
$$

yields an exact sequence

$$
N=\bigoplus_{C \in[u], C \neq D} M_{C}
$$

$$
0 \rightarrow N \oplus M_{D \backslash E(d)} \rightarrow N \oplus M_{D} \rightarrow M_{E(d)} \rightarrow 0 .
$$

These modules are $\mathbf{Z}$-free, since $M$ is projective. Using (2.7) and (1.5) we get an isomorphism of fields

$$
l\left(N \oplus M_{D \backslash E(d)} \oplus M_{E(d)}\right)^{\pi} \cong l\left(N \oplus M_{D}\right)^{\pi}
$$

over $l^{n}$. Because of $(2.9)$ this is exactly the same as $l(M(u))^{\pi} \cong l(M(v))^{\pi}$ over $l^{\pi}$.

Proof of (2.4). Let $i(m), w(m) \in G(m)$ be as in the proof of $(2.10)$. Then

$$
\begin{aligned}
M(i(m)) & =\bigoplus_{d / m} M / \Phi_{d}(\tau) M \cong \underset{\rho}{\oplus} F_{\rho}(M), \\
M(w(m)) & =M /\left(\tau^{m}-1\right) M=M .
\end{aligned}
$$

So (2.4) follows from (2.11) if we put $u=i(m)$ and $v=w(m)$. 
Remark. Theorem (2.4) can be generalized to the case $M$ is permutation-projective over $\pi$. The only modification in the proof is that for $C \subset E(m)$ the module $M_{C}$ has to be redefined as follows:

$$
M_{C}=M /\left\{x \in M \mid \exists k \in \mathbf{Z}, k \neq 0: k \cdot x \in \Phi_{C}(\tau) \cdot M\right\},
$$

and that $C^{\prime}$ in (2.8) must be equal to $E(d)$, for some $d \in E(m)$.

\section{The Modules $I_{q}$ and $J_{q}$}

Let $p$ be a prime number, and let $q=p^{s}$ be a power of $p$, with $s \geqq 1$. In this section $l$ denotes a field of characteristic $\neq p$ which contains a primitive $q$-th root of unity $\zeta_{q}$, and $\pi$ is a finite abelian group of automorphisms of $l$. We put $k=l^{\pi}$ and $\pi(q)=\left\{\sigma \in \pi \mid \sigma\left(\zeta_{q}\right)=\zeta_{q}\right\}=\operatorname{Gal}\left(l / k\left(\zeta_{q}\right)\right)$. Let $\rho(q)=\operatorname{Gal}\left(k\left(\zeta_{q}\right) / k\right)=\pi / \pi(q)$. The map $\pi \rightarrow(\mathbf{Z} / q \mathbf{Z})^{*}$, which sends $\tau$ to $(t \bmod q)$ if $\tau\left(\zeta_{q}\right)=\zeta_{q}^{t}$, gives rise to an injective group homomorphism $\phi_{q}: \rho(q) \rightarrow(\mathbf{Z} / q \mathbf{Z})^{*}$. This map makes $\mathbf{Z} / q \mathbf{Z}$ into a $\rho(q)$-module and hence into a $\pi$-module.

We consider first the case when $\rho(q)$ is non-cyclic, and afterwards the case when $\rho(q)$ is cyclic.

So assume that $\rho(q)$ is non-cyclic. Then $q$ is divisible by 8 . Put $C(q)=(\mathbf{Z} / q \mathbf{Z}) \backslash\{0\}$, and let $\mathbf{Z}^{C(q)}$ be a free abelian group of rank $q-1$ with $\mathbf{Z}$-basis $\left\{e_{c} \mid c \in C(q)\right\}$. We make $\mathbf{Z}^{C(q)}$ into a $\rho(q)$-module by $\sigma\left(e_{c}\right)=e_{\sigma c}$, for $\sigma \in \rho(q)$ and $c \in C(q)$. Then the group homomorphism $\mathbf{Z}^{(c)} \rightarrow \mathbf{Z} / q \mathbf{Z}$, mapping $e_{c}$ to $c$ for $c \in C(q)$, is $\rho(q)$-linear, and we call its kernel $I_{q}$. So there is an exact sequence of $\rho(q)$-modules

$$
0 \rightarrow I_{q} \rightarrow \mathbf{Z}^{C(q)} \rightarrow \mathbf{Z} / q \mathbf{Z} \rightarrow 0 .
$$

(3.1) Proposition. For every subgroup $\pi^{\prime} \subset \pi$ we have $H^{1}\left(\pi^{\prime}, I_{q}\right)=0$.

Proof. Obvious from the exact sequence of cohomology.

(3.2) Proposition. For some subgroup $\pi^{\prime} \subset \pi$ we have $\hat{H}^{-1}\left(\pi^{\prime}, I_{q}\right) \neq 0$.

Proof (sketch). Since $I_{q}$ is torsion free, we may assume $\pi=\rho(q)$. We assumed that $\pi$ is non-cyclic, so there is a subgroup $\pi^{\prime}$ with $\phi_{q}\left[\pi^{\prime}\right]=$ $\{1, u-1, u+1,-1\} \subset \mathbf{Z} / q \mathbf{Z}$, where $u=\frac{1}{2} q$. We are going to prove $\hat{H}^{-1}\left(\pi^{\prime}, I_{q}\right) \cong \mathbf{Z} / 2 \mathbf{Z}$.

Put $C=\{1, u-1, u, u+1,-1\} \subset C(q) \subset \mathbf{Z} / q \mathbf{Z}$. Then $\mathbf{Z}^{C}$ is a sub- $\pi^{\prime}-$ module of $Z^{C(q)}$ in an obvious way, and restricting the $\operatorname{map} \mathbf{Z}^{C(q)} \rightarrow \mathbf{Z} / q \mathbf{Z}$ to $\mathbf{Z}^{C}$ we get an exact sequence of $\pi^{\prime}$-modules

$$
0 \rightarrow M \rightarrow \mathbf{Z}^{C} \rightarrow \mathbf{Z} / q \mathbf{Z} \rightarrow 0
$$

where $M=\mathbf{Z}^{C} \cap I_{q}$. The exact sequence of cohomology easily yields $H^{1}\left(\pi^{\prime \prime}, M\right)=0$ for all five subgroups $\pi^{\prime \prime} \subset \pi^{\prime}$, and an explicit computation 
inside $M$ shows $\hat{H}^{-1}\left(\pi^{\prime}, M\right) \cong \mathbf{Z} / 2 \mathbf{Z}$. By diagram chasing one gets an exact sequence

$$
0 \rightarrow M \rightarrow I_{q} \rightarrow \mathbf{Z}^{C(q)}-C \rightarrow 0,
$$

which splits by (1.2). Using (1.1) we find $\hat{H}^{-1}\left(\pi^{\prime}, I_{q}\right) \cong \mathbf{Z} / 2 \mathbf{Z}$, as required.

For the remainder of this section we assume $\rho(q)$ is cyclic. The ring homomorphism $\mathbf{Z}[\rho(q)] \rightarrow \mathbf{Z} / q \mathbf{Z}$ induced by $\phi_{q}$ is $\rho(q)$-linear, and we call its kernel $J_{q}$. So there is an exact sequence of $\rho(q)$-modules

$$
0 \rightarrow J_{q} \rightarrow \mathbf{Z}[\rho(q)] \rightarrow \mathbf{Z} / q \mathbf{Z} \rightarrow 0 .
$$

(3.3) Proposition [10]. Let $\rho(q)$ be cyclic. Then $J_{q}$ is a projective $\rho(q)$ module except if (3.4) holds:

$$
q \equiv 0 \bmod 4 \text { and } \phi_{q}[\rho(q)]=\{+1,-1\} \subset(\mathbf{Z} / q \mathbf{Z})^{*} .
$$

Proof. Suppose (3.4) does not hold. Let $\rho=\phi_{q}[\rho(q)] \subset(\mathbf{Z} / q \mathbf{Z})^{*}$ and $n=|\rho|=|\rho(q)|$. Let $\rho_{1} \subset(\mathbf{Z} / p q \mathbf{Z})^{*}$ be the inverse image of $\rho$ under the canonical map $(\mathbf{Z} / p q \mathbf{Z})^{*} \rightarrow(\mathbf{Z} / q \mathbf{Z})^{*}$. Then $\rho_{1}$ has order $n p$, and we claim that $\rho_{1}$ is cyclic.

Suppose, in fact, that $\rho_{1}$ is non-cyclic. Then $(\mathbf{Z} / p q \mathbf{Z})^{*}$ is non-cyclic, so $p=2$ and $q \equiv 0 \bmod 4$. Moreover, $(-1 \bmod p q) \in \rho_{1}$, so $-1 \in \rho$. But the only cyclic subgroup of $(\mathbf{Z} / q \mathbf{Z})^{*}$ containing -1 is $\{+1,-1\}$, so $\rho=\{+1,-1\}$. Hence (3.4) holds, contradicting our assumption. We conclude that $\rho_{1}$ is cyclic.

Choose $t \in \mathbf{Z}$ such that $(t \bmod p q)$ generates $\rho_{1}$. Since $\left|\rho_{1}\right|>n$, we have $t^{n} \neq 1 \bmod p q$. Clearly, $(t \bmod q)$ generates $\rho$, so $t^{n} \equiv 1 \bmod q$. Hence $t^{n}-1=a \cdot q$, where $a$ and $q$ are relatively prime.

Let $\tau \in \rho(q)$ be such that $\phi_{q}(\tau)=(t \bmod q)$. Then $\tau$ generates $\rho(q)$, and the $\mathbf{Z}[\rho(q)]$-ideal $J_{q}$ is generated by $\tau-t$ and $q$. Denote by $M$ the $\mathbf{Z}[\rho(q)]$-ideal generated by $\tau-t$ and $a$. Then $J_{q}+M=\mathbf{Z}[\rho(q)]$, so $J_{q} \cap M=J_{q} \cdot M$. Hence we have an exact sequence of $\mathbf{Z}[\rho(q)]$-modules

$$
0 \rightarrow J_{q} \cdot M \rightarrow J_{q} \oplus M \rightarrow \mathbf{Z}[\rho(q)] \rightarrow 0
$$

where the map $J_{q} \oplus M \rightarrow \mathbf{Z}[\rho(q)]$ is defined by $(j, m) \mapsto j-m$. The ideal $J_{q} \cdot M$ is generated by the four elements $\left\{(\tau-t)^{2}, a(\tau-t), q(\tau-t), a q\right\}$ where $a q=t^{n}-\tau^{n}$. It follows that $J_{q} \cdot M=\mathbf{Z}[\rho(q)] \cdot(\tau-t)$ is a free $\mathbf{Z}[\rho(q)]$ module, and since the above sequence splits we find that $J_{q}$ is $\rho(q)$ projective.

Remark. If (3.4) holds, then $J_{q}$ is not projective. In fact, suppose $q \equiv 0 \bmod 4$ and $\rho(q)=\{1, \tau\}$, where $\phi_{q}(\tau)=-1$. Then $J_{q}$ has a $\mathbf{Z}$-basis $\left\{1+\tau, \frac{1}{2} q-\frac{1}{2} q \cdot \tau\right\}$, so $J_{q} \cong \mathbf{Z} \oplus \mathbf{Z}^{\prime}$; here $\rho(q)$ acts trivially on $\mathbf{Z}$, while the $\rho(q)$-module $\mathbf{Z}^{\prime}$ has underlying abelian group $\mathbf{Z}$ and $\rho(q)$-action $\tau \cdot m=-m$, for $m \in \mathbf{Z}$.

21 Inventiones math. Vol. 25 
(3.5) Proposition. Suppose $q$ is a power of 2 and $\rho(q)$ is cyclic. Then $l\left(J_{q}\right)^{\pi}$ is rational over $l^{\pi}$.

Proof. Replacing $l$ by $l^{\pi(q)}$ we may assume $\pi=\rho(q)$. Suppose first that (3.4) holds. Then by the above remark $l\left(J_{q}\right)=l(x, y)$, where $\tau(x)=x$ and $\tau(y)=y^{-1}$; here $\tau$ denotes the non-trivial element of $\pi$. Choose $\alpha \in l$ with $\tau(\alpha) \neq \alpha$. Then $l(x, y)^{\pi}=l^{\pi}(x, z)$, where $z=(\alpha y+\tau(\alpha)) /(y+1)$. So in this case $l\left(J_{q}\right)^{\pi}$ is rational over $l^{\pi}$.

Suppose now that (3.4) does not hold. By (3.3), the $\pi$-module $J_{q}$ is projective, so we are in a position to apply (2.6). Hence we need only check that $F_{\rho}\left(J_{q}\right)$ is $\mathbf{Z}(\rho)$-free for every factor group $\rho$ of $\pi$.

So let $\rho \neq\{1\}$ be a factor group of $\pi$ of order $2^{r}$. Since $J_{q}$ has index $q$ in $\mathbf{Z}[\pi]$, it follows from (2.2) that $F_{\rho}\left(J_{q}\right)$ may be considered as a submodule of 2-power index in $F_{p}(\mathbf{Z}[\pi]) \cong \mathbf{Z}(\rho)$. But $\mathbf{Z}(\rho) \cong \mathbf{Z}\left[\zeta_{2^{r}}\right]$, and every ideal of 2-power index in $Z\left[\zeta_{2 r}\right]$ is generated by the corresponding power of $1-\zeta_{2 r}$, and is therefore free. It follows that $F_{\rho}\left(J_{q}\right)$ is $\mathbf{Z}(\rho)$-free, as required

If $K$ is a subfield of $l$ which is a cyclic extension of $k$, then $\operatorname{Gal}(K / k)$ is a cyclic factor group of $\pi$, and we will write $F_{K}$ instead of $F_{\mathrm{Gal}(K / k)}$.

(3.6) Proposition. Suppose $q$ is odd. Let $K$ be an intermediate field $k \subset K \subset l$ such that $\rho_{K}=\mathrm{Gal}(K / k)$ is cyclic. Let $\mathbf{a}_{K}(-)$ be as in the introduction. Then

$$
\begin{aligned}
& F_{\boldsymbol{K}}\left(J_{q}\right) \cong \mathfrak{a}_{K}(\mathbf{Z} / q \mathbf{Z}) \quad \text { as } \mathbf{Z}\left(\rho_{K}\right) \text {-modules if } K \subset k\left(\zeta_{q}\right), \\
& F_{\boldsymbol{K}}\left(J_{q}\right)=0 \quad \text { if } K \nsubseteq k\left(\zeta_{q}\right) .
\end{aligned}
$$

This is proved after the proof of (3.7).

(3.7) Lemma. Let $q=p^{s}$ be odd, let $\tau$ be a generator of $\rho(q)=\pi / \pi(q)=$ $\mathrm{Gal}\left(k\left(\zeta_{q}\right) / k\right)$, and choose $t \in \mathbf{Z}$ such that $\tau\left(\zeta_{q}\right)=\zeta_{q}$. We denote the order of $(t \bmod p) \in(\mathbf{Z} / p \mathbf{Z})^{*}$ by $f$, and we put $p^{r}=$ g.c.d. $\left(q, t^{\mathcal{S}}-1\right)$; here $r \in \mathbf{Z}$ and $1 \leqq r \leqq s$.

In this situation, any intermediate field $k \subset K \subset k\left(\zeta_{q}\right)$ is uniquely determined by its degree $[K: k]$ over $k$. Moreover, if $K$ is such a field, then $\rho_{K}=\mathrm{Gal}(K / k)$ is a cyclic group, generated by the image $\tau_{K}$ of $\tau$ in $\rho_{K}$. We have:

(i) if $K=k\left(\zeta_{p}\right)$, then $K=k\left(\zeta_{p^{*}}\right)$ for all $1 \leqq i \leqq r$, the degree $[K: k]$ equals $f$, and $F_{K}\left(J_{q}\right)$ is, as a $\mathbf{Z}\left(\rho_{K}\right)$-module, isomorphic to the $r$-th ideal power of the $\mathbf{Z}\left(\rho_{K}\right)$-ideal generated by $p$ and $\tau_{K}-t \in \mathbf{Z}\left(\rho_{K}\right)$;

(ii) if $K=k\left(\zeta_{p^{i}}\right)$ with $r<i \leqq s$, then $[K: k]=f \cdot p^{i-r}$, and $F_{K}\left(J_{q}\right)$ is, as a $\mathbf{Z}\left(\rho_{K}\right)$-module, isomorphic to the $\mathbf{Z}\left(\rho_{K}\right)$-ideal generated by $p$ and $\tau_{K}-t$;

(iii) for all other $K \subset k\left(\zeta_{q}\right)$, we have $F_{K}\left(J_{q}\right) \cong \mathbf{Z}\left(\rho_{K}\right)$ as $\mathbf{Z}\left(\rho_{K}\right)$-modules. 
(3.8) Lemma. For $m \in \mathbf{Z}$, let ord $(m)$ denote the number of factors $p$ in $m$. Let $t$ and $f$ be as in (3.7). Then:

(i) $\operatorname{ord}\left(\Phi_{f}(t)\right)=\operatorname{ord}\left(t^{f}-1\right)>0$, $\operatorname{ord}\left(\Phi_{f p^{i}}(t)\right)=1$ for all $i \in \mathbf{Z}, i>0$, $\operatorname{ord}\left(\Phi_{d}(t)\right)=0$ for all other $d \in \mathbf{Z}, d>0$.

(ii) $\operatorname{ord}\left(t^{m}-1\right)=0$ if $m \in \mathbf{Z}, m>0$ and $m \neq 0 \bmod f$, $\operatorname{ord}\left(t^{m}-1\right)=\operatorname{ord}\left(t^{f}-1\right)+\operatorname{ord}(m)$ if $m \in \mathbf{Z}, m>0$ and $m \equiv 0 \bmod f$.

Proof of (3.8). See [1, Lemma 1]. D

Proof of (3.7). Since $k\left(\zeta_{q}\right)$ is a cyclic extension of $k$, it is clear that an intermediate field $K$ is determined by its degree over $k$, and that $\rho_{\mathbf{K}}$ is generated by the image of $\tau$.

Let $1 \leqq i \leqq s$. By Galois theory, $\left[k\left(\zeta_{p^{i}}\right): k\right]$ is the smallest positive integer $m$ for which $\tau^{m}\left(\zeta_{p^{i}}\right)=\zeta_{p^{i}}$, i.e., for which $t^{m}-1 \equiv 0 \bmod p^{i}$. From (3.8) (ii) it follows then that $\left[k\left(\zeta_{p^{i}}\right): k\right]=f$ if $1 \leqq i \leqq r$, and $\left[k\left(\zeta_{p^{i}}\right): k\right]=f \cdot p^{i-\text {, }}$ if $r<i \leqq s$. This proves the statements concerning the degrees $\left[k\left(\zeta_{p^{i}}\right): k\right]$. In particular, $\left[k\left(\zeta_{q}\right): k\right]=f \cdot p^{s-r}$.

Now let $k \subset K \subset k\left(\zeta_{q}\right)$ be such that $[K: k]=d$, where $d \mid f \cdot p^{s-r}$. Tensoring the exact sequence defining $J_{q}$ with $\mathbf{Z}\left(\rho_{K}\right)$ over $\mathbf{Z}[\rho(q)]$, we find an exact sequence of $\mathbf{Z}\left(\rho_{K}\right)$-modules

$$
J_{q} \otimes_{\rho(q)} \mathbf{Z}\left(\rho_{K}\right) \rightarrow \mathbf{Z}[\rho(q)] \otimes_{\rho(q)} \mathbf{Z}\left(\rho_{K}\right) \rightarrow(\mathbf{Z} / q \mathbf{Z}) \otimes_{\rho(q)} \mathbf{Z}\left(\rho_{K}\right) \rightarrow 0
$$

Since $J_{q}$ is projective, the first two modules in this sequence are $F_{K}\left(J_{q}\right)$ and $F_{K}(\mathbf{Z}[\rho(q)]) \cong \mathbf{Z}\left(\rho_{K}\right)$, and the first arrow is injective by (2.2). Using $\mathbf{Z}\left(\rho_{K}\right)=\mathbf{Z}[\rho(q)] / \Phi_{d}(\tau) \mathbf{Z}[\rho(q)]$ we find for the cokernel:

$$
\begin{aligned}
(\mathbf{Z} / q \mathbf{Z}) \otimes_{\rho(q)} \mathbf{Z}\left(\rho_{K}\right) & \cong(\mathbf{Z} / q \mathbf{Z}) / \Phi_{d}(\tau) \cdot(\mathbf{Z} / q \mathbf{Z}) \\
& =\mathbf{Z} /\left(q \cdot \mathbf{Z}+\Phi_{d}(t) \cdot \mathbf{Z}\right)
\end{aligned}
$$

since $\tau$ acts on $\mathbf{Z} / q \mathbf{Z}$ as multiplication by $t$.

Summarizing, we have an exact sequence of $Z\left(\rho_{K}\right)$-modules

$$
0 \rightarrow F_{K}\left(J_{q}\right) \rightarrow \mathbf{Z}\left(\rho_{K}\right) \rightarrow \mathbf{Z} /\left(q \cdot \mathbf{Z}+\Phi_{d}(t) \cdot \mathbf{Z}\right) \rightarrow 0
$$

where the map $\mathbf{Z}\left(\rho_{\boldsymbol{K}}\right) \rightarrow \mathbf{Z} /\left(q \cdot \mathbf{Z}+\Phi_{d}(t) \cdot \mathbf{Z}\right)$ sends $\tau_{\boldsymbol{K}}$ to the residue class of $t$.

In case (iii) we have g.c.d. $\left(q, \Phi_{d}(t)\right)=1$, by $(3.8)(\mathrm{i})$, so $F_{K}\left(J_{q}\right)$ is isomorphic to $Z\left(\rho_{K}\right)$. In case (ii), we have g.c.d. $\left(q, \Phi_{d}(t)\right)=p$, by $(3.8)(\mathrm{i})$, so $F_{K}\left(J_{q}\right)$ is isomorphic to $\left(p, \tau_{K}-t\right) \subset \mathbf{Z}\left(\rho_{K}\right)$. Finally, in case (i) we have g.c.d. $\left(q, \Phi_{d}(t)\right)=p^{r}$, so $\mathbf{Z}\left(\rho_{K}\right) / F_{K}\left(J_{q}\right) \cong \mathbf{Z} / p^{r} \mathbf{Z}$ is a local ring. Therefore $F_{K}\left(J_{q}\right)$ is an ideal power of $\left(p, \tau_{K}-t\right)$, and computing norms we find that the exponent has to be $r$. 
Proof of (3.6). For $K+k\left(\zeta_{q}\right)$ we have $F_{K}\left(J_{q}\right)=0$ by (2.1). Therefore it suffices to consider subfields $K \subset k\left(\zeta_{q}\right)$. These fields are described in (3.7), and for each of them $F_{K}\left(J_{q}\right)$ is computed. Comparing the outcome with the definition of $\mathfrak{a}_{K}(\mathbf{Z} / q \mathbf{Z})$ (see introduction) one finds that $F_{K}\left(J_{q}\right) \cong \mathfrak{a}_{K}(\mathbf{Z} / q \mathbf{Z})$, as required.

\section{A Reduction}

Let $k$ be a field, and $A$ a finite abelian group. The field $k_{A}$ has been defined in the introduction. We write $A \cong P \oplus B$, such that $|B| \neq 0$ mod char $(k)$ while $|P|$ is a power of $\operatorname{char}(k)$.

(4.1) Proposition. The field $k_{A}$ is $k$-isomorphic to a rational field extension of $k_{B}$.

Before proving (4.1) we state two lemmas.

(4.2) Lemma. Let $K_{0} \subset K_{1} \subset \cdots \subset K_{d}$ be a chain of fields of characteristic $p \neq 0$, such that for each $i$ with $1 \leqq i \leqq d$ there is an element $u_{i} \in K_{i}$ such that $K_{i}=K_{i m 1}\left(u_{i}\right)$. Let $P$ be a finite p-group of field automorphisms of $K_{d}$ such that

(i) the action of $P$ on $K_{0}$ is trivial;

(ii) $\sigma\left(u_{i}\right)-u_{i} \in K_{i-1}$ for all $\sigma \in P$ and $1 \leqq i \leqq d$.

Then $K_{d}^{P}=K_{0}\left(z_{1}, \ldots, z_{d}\right)$ for some $z_{1}, \ldots, z_{d} \in K_{d}$.

Proof of (4.2). This lemma is Satz 2 of [16]. For a short proof, see [33]

(4.3) Lemma. Let $K$ be a field of characteristic $p \neq 0$, and let $P$ be a finite p-group. Let $M$ be a nonzero $K[P]$-module. Then $M^{P} \neq 0$.

Proof. See [42, Ch. IX, Th. $2 ; 6$, Ch. IV, $\S 9 ; 33]$. ]

Proof of (4.1). Put $p=\operatorname{char}(k)$. Clearly we may assume $p \neq 0$.

We denote by $V$ the $k$-vector space inside $k\left(\left\{x_{g} \mid g \in A\right\}\right)$ generated by $\left\{x_{g} \mid g \in A\right\}$. Clearly, $V$ is a $k[A]$-module isomorphic to the left module $k[A]$. Let $W \subset V$ be the subspace $W=V^{P}$. This is a $k[B]$-module isomorphic to $k[B]$. Therefore, to prove (4.1) it suffices to show that $k(V)^{A}$ is rational over $k(W)^{B}$; here $k(W)$ denotes the field generated by $k$ and $W$ inside $k(V)=k\left(\left\{x_{g} \mid g \in A\right\}\right)$. The codimension of $W$ in $V$ is denoted by $d$; we have $d=|A|-|B|$.

By $U$ we denote the $k(W)$-vector space spanned by $V$ inside $k(V)$. It is easy to see that $U$ has dimension $d+1$ over $k(W)$, that $1 \in U$, and that $B$ acts semilinearly on $U$. Put $T=U^{B}$. Then from (1.3) it follows that $T$ is a $(d+1)$-dimensional vector space over $k(W)^{B}$ with $1 \in T$.

The definition of $T$ implies $\sigma T=T$ for $\sigma \in P$, so $T$ is a $k(W)^{B}[P]$ module. We choose a sequence of $k(W)^{B}[P]$-submodules $Y_{i}$ of $T$, for 
$0 \leqq i \leqq d$, such that $Y_{0}=k(W)^{B} \cdot 1$ and such that for each $i$ with $1 \leqq i \leqq d$ we have:

$Y_{i-1} \subset Y_{i}$, and $Y_{i} / Y_{i-1}$ is a one-dimensional vector space over $k(W)^{B}$ on which $P$ acts trivially.

Such a sequence $\left(Y_{i}\right)_{i=0}^{d}$ is easily constructed by induction on $i$ : just apply (4.3) to $M=T / Y_{i-1}$ to find $Y_{i}$. Of course, $Y_{d}=T$.

Let $u_{i} \in Y_{i}$ be such that $Y_{i}$ as a $k(W)^{B}$-vector space is generated by $Y_{i-1}$ and $u_{i}$, for $1 \leqq i \leqq d$. Let $K_{i}$ be the field generated by $k(W)^{B}$ and $Y_{i}$, for $0 \leqq i \leqq d$. Then $K_{0}=k(W)^{B}$ and we claim

$$
K_{d}=k(V)^{B} .
$$

Assume (4.4) for a moment. The conditions of (4.2) are satisfied, by construction, so

$$
K_{d}^{P}=K_{0}\left(z_{1}, \ldots, z_{d}\right)
$$

for some $z_{1}, \ldots, z_{d} \in K_{d}$, or, what is the same,

$$
k(V)^{A}=\left(k(V)^{B}\right)^{P}=k(W)^{B}\left(z_{1}, \ldots, z_{d}\right) .
$$

Counting transcendence degrees we conclude that $k(V)^{A}$ is rational over $k(W)^{B}$, as required.

It remains to prove (4.4). By definition,

$$
K_{d}=k(W)^{B}(T)=k(W)^{B}\left(U^{B}\right),
$$

so the inclusion $K_{d} \subset k(V)^{B}$ is obvious. We prove equality by a degree calculation.

Using (1.3) we choose a $B$-invariant $k(W)$-basis $\left\{b_{0}, \ldots, b_{d}\right\}$ for $U$. Then $\left\{b_{0}, \ldots, b_{d}\right\}$ is a $k(W)^{B}$-basis for $U^{B}$ so

$$
K_{d}=k(W)^{B}\left(b_{0}, \ldots, b_{d}\right)
$$

while

Therefore

$$
k(V)=k(W)(U)=k(W)\left(b_{0}, \ldots, b_{d}\right) .
$$

and since

$$
\left[k(V): K_{d}\right] \leqq\left[k(W): k(W)^{B}\right]=|B|,
$$

$$
\left[k(V): k(V)^{B}\right]=|B|
$$

it follows that $K_{d}=k(V)^{B}$. This completes the proof of (4.1).

\section{Proof of the Main Theorem}

Let $k$ be a field and $A$ a finite abelian group. We write $A \cong P \oplus B$ as in the preceding section. By $e$ we denote the exponent of $B$, and we put $l=k\left(\zeta_{e}\right)$. The Galois group of $l$ over $k$ is called $\pi$. As is well known, 
the character group $D=\operatorname{Hom}\left(B, l^{*}\right)$ is, as an abelian group, isomorphic to $B$ (non-canonically). We make $D$ into a $\pi$-module by $(\sigma d)(g)=\sigma(d(g))$ for $\sigma \in \pi, d \in D$ and $g \in B$. Let $Z^{D}$ be a free abelian group with $\left\{e_{d} \mid d \in D\right\}$ as a $\mathbf{Z}$-basis, and make $\mathbf{Z}^{D}$ into a permutation module over $\pi$ by $\sigma e_{d}=e_{\sigma d}$, for $\sigma \in \pi$ and $d \in D$. The group homomorphism $\mathbf{Z}^{D} \rightarrow D$ sending $e_{d}$ to $d$, for $d \in D$, is $\pi$-linear, and we call its kernel $J$. So we have an exact sequence of $\pi$-modules

$$
0 \rightarrow J \rightarrow \mathbf{Z}^{D} \rightarrow D \rightarrow 0 .
$$

(5.1) Proposition $[12,31]$. The fields $k_{B}$ and $l(J)^{\pi}$ are isomorphic over $k=l^{n}$.

Proof. Let $l(x)=l\left(\left\{x_{g} \mid g \in B\right\}\right)$ and $k(x)=k\left(\left\{x_{g} \mid g \in B\right\}\right)$. First we describe $i_{B}=l(x)^{B}$.

For $d \in D$, let

$$
y_{d}=\left(\sum_{g \in B} d(g)^{-1} \cdot x_{g}\right) \in l(x) .
$$

Then $l(x)=l\left(\left\{y_{d} \mid d \in D\right\}\right)$, and the action of $B$ on $l\left(\left\{y_{d} \mid d \in D\right\}\right)$ is given by

$$
g\left(y_{d}\right)=d(g) \cdot y_{d}, \quad \text { for } g \in B \text { and } d \in D .
$$

Let $F \subset l(x)^{*}$ be the multiplicative subgroup generated by $\left\{y_{d} \mid d \in D\right\}$. Clearly, $F$ is Z-free of rank $|D|=|B|$. Define the homomorphism $\phi: F \rightarrow D$ by sending $y_{d}$ to $d$, for $d \in D$. Then

$$
g(y)=\phi(y)(g) \cdot y \quad \text { for } y \in F \text { and } g \in B .
$$

So if $y \in \operatorname{ker}(\phi)$ then $g(y)=y$ for all $y \in B$, i.e. $y \in l_{B}$. This means

$$
l(\operatorname{ker}(\phi)) \subset l_{B} \subset l(x)=l(F) .
$$

The index of $\operatorname{ker}(\phi)$ in $F$ equals $|D|$. Therefore we find, by extracting roots successively:

$$
[l(F): l(\operatorname{ker}(\phi))] \leqq|D| .
$$

But $\left[l(F): l_{B}\right]=|D|$ by Galois theory, so we conclude $l(\operatorname{ker}(\phi))=l_{B}$. Since a $\mathbf{Z}$-basis for $\operatorname{ker}(\phi)$ is algebraically independent over $l$, the field $l(\operatorname{ker}(\phi))$ is isomorphic to the field of fractions of the group ring of $\operatorname{ker}(\phi)$ over $l$. This removes a slight ambiguity in our notations, cf. Section 1 .

Next we let come in $k$. We let $\pi$ act on $l(x) \cong l \bigotimes_{k} k(x)$ via the first factor. Then the actions of $\pi$ and $B$ on $l(x)$ commute, so

One easily checks

$$
k_{B}=\left(l(x)^{\pi}\right)^{B}=\left(l(x)^{B}\right)^{\pi}=\left(l_{B}\right)^{\pi} .
$$

$$
\sigma\left(y_{d}\right)=y_{\sigma d} \quad \text { for } \sigma \in \pi \text { and } d \in D,
$$


so $F$ is a sub- $\pi$-module of $l(x)^{*}$, and $F \cong Z^{D}$. The map $\phi: F \rightarrow D$ is $\pi$-linear, so $\operatorname{ker}(\phi)$ is a sub- $\pi$-module of $F$, and clearly $\operatorname{ker}(\phi) \cong J$. It follows that there is an $l$-isomorphism of fields $l_{B}=l(\operatorname{ker}(\phi)) \cong l(J)$ which respects the action of $\pi$. Hence there is an isomorphism

$$
k_{B}=\left(l_{B}\right)^{\pi} \cong l(J)^{\pi}
$$

over $k=l^{\pi}$, as required. $\quad$

We write

$$
B \cong \underset{q}{(})(\mathbf{Z} / q \mathbf{Z})^{n(q)} \quad \text { (as abelian groups) }
$$

with non-negative integers $n(q)$, where $q$ ranges over the set of prime powers $>1$. We define the $\pi$-modules $I_{1}, I_{2}$ and $I_{3}$ by

$$
\begin{aligned}
& I_{1}=\underset{q . q \text { is odd }}{\oplus} J_{q}^{n(q)}, \\
& I_{2}=\underset{q, \rho(q) \text { is non-cyclic }}{\oplus} I_{q}^{n(q)}, \\
& I_{3}=\underset{q, q \text { is eqen, } \rho(q) \text { is cyclic }}{\oplus} J_{q}^{n(q)} .
\end{aligned}
$$

(See Section 3 for the definitions of $I_{q}, J_{q}$ and $\rho(q)$ ). Finally, we put

$$
I=I_{1} \oplus I_{2} .
$$

(5.2) Proposition. The field $l(J)^{n}$ is $l^{\pi}$-isomorphic to a rational extension of $l(I)^{\pi}$.

Proof. By a $\pi$-set we mean a set $E$ on which $\pi$ acts as a group of permutations (the action need not be faithful). The corresponding permutation module is denoted by $\mathbf{Z}^{E}$. A subset $E^{\prime}$ of a $\pi$-set is called a $\pi$-subset if $\sigma\left(e^{\prime}\right) \in E^{\prime}$ for all $\sigma \in \pi$ and $e^{\prime} \in E^{\prime}$.

The decomposition

$$
B \cong \bigoplus \Theta_{c}(\mathbf{Z} / q \mathbf{Z})^{n(q)}
$$

gives rise to a decomposition of $\pi$-modules

$$
D \cong \Theta_{q}(\mathbf{Z} / q \mathbf{Z})^{n(q)},
$$

each direct summand $\mathbf{Z} / q \mathbf{Z}$ being a $\pi$-module as described in Section 3 .

We first consider a direct summand $Z / q Z$ for which $p(q)$ is noncyclic. If $\mathbf{Z} / q \mathbf{Z} \rightarrow D$ is an injective $\pi$-homomorphism identifying $\mathbf{Z} / q \mathbf{Z}$ with one of the direct summands, then the resulting injection of $\pi$-sets $C(q) \subset \mathbf{Z} / q \mathbf{Z} \rightarrow D$ (see Section 3 for the definition of $C(q)$ ) gives rise to a $\pi$-linear map $\mathbf{Z}^{\mathbf{C}(q)} \rightarrow \mathbf{Z}^{D}$. It is easily checked that the following diagram 
with exact rows is then commutative:

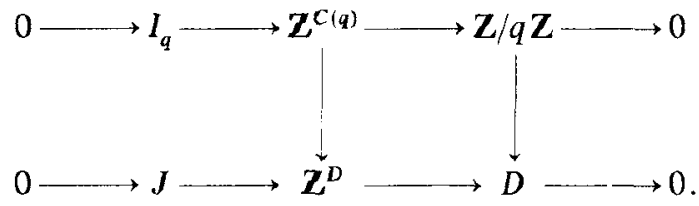

Analogously, if $\rho(q)$ is cyclic, and we have a $\pi$-homomorphism $\mathbf{Z} / q \mathbf{Z} \rightarrow D$ which identifies $\mathbf{Z} / q \mathbf{Z}$ with one of the direct summands, then an injective map of $\pi$-sets $\rho(q) \rightarrow(\mathbf{Z} / q \mathbf{Z})^{*} \subset \mathbf{Z} / q \mathbf{Z} \rightarrow D$ is induced (here the map $\rho(q) \rightarrow(\mathbf{Z} / q \mathbf{Z})^{*}$ is the map $\phi_{q}$ defined in Section 3$)$. The resulting $\pi$ homomorphism $\mathbf{Z}[\rho(q)] \cong \mathbf{Z}^{(q)} \rightarrow \mathbf{Z}^{D}$ then makes the following diagram with exact rows commutative:

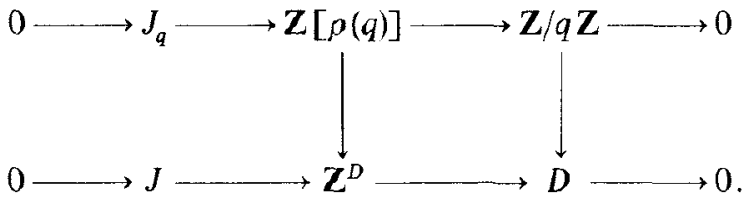

So with each direct summand $\mathbf{Z} / q \mathbf{Z}$ of $D$ we have associated a diagram, and all these diagrams have the same second row. Taking the direct sum of all first rows we find the commutative diagram with exact rows

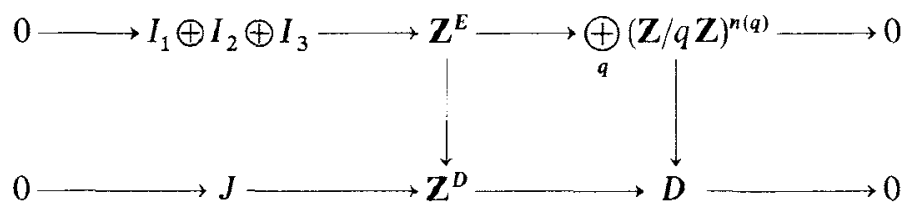

where $E$ is some $\pi$-set which is a disjoint union of $\pi$-sets of the form $C(q)$ and $\rho(q)$, with certain multiplicities. Since $0 \notin C(q) \subset \mathbf{Z} / q \mathbf{Z}$ and $0 \notin \phi_{q}[\rho(q)] \subset \mathbf{Z} / q \mathbf{Z}$, the images of these $\pi$-sets in $D$ do not overlap. This means that $E$ may be considered as a $\pi$-subset of $D$, and that the $\operatorname{map} \mathbf{Z}^{E} \rightarrow \mathbf{Z}^{D}$ is injective and has a cokernel $N$ which is itself a permutation module over $\pi$. Since the second vertical arrow in the above diagram is an isomorphism, we get an exact sequence of $\pi$-modules

$$
0 \rightarrow I \oplus I_{3} \rightarrow J \rightarrow N \rightarrow 0
$$

in which $N$ is a permutation module. From (1.6) it follows that $l(J)^{\pi}$ is $l^{\pi}$-isomorphic to a rational field extension of $l\left(I \oplus I_{3}\right)^{\pi}$. Applying (3.5) we find that $l\left(I \oplus I_{3}\right)^{\pi}$ is rational over $l(I)^{\pi}$. This proves (5.2).

(5.3) Proposition. The field $k_{A}$ is $k$-isomorphic to a rational field extension of $l(I)^{\pi}$. 
Proof. Combine (4.1), (5.1) and (5.2).

(5.4) Proposition. For every subgroup $\pi^{\prime} \subset \pi$ we have $H^{1}\left(\pi^{\prime}, I\right)=0$.

Proof. This follows from (3.1), (3.3) and the definition of $I$.

(5.5) Proposition. Let $k \subset K \subset l$ be an intermediate field such that $p_{K}=$ $\mathrm{Gal}(K / k)$ is cyclic. Then $F_{K}\left(I_{1}\right)$ is $\mathbf{Z}\left(\rho_{K}\right)$-free if and only if the $\mathbf{Z}\left(\rho_{K}\right)$-ideal $a_{K}(A)$ is principal.

Proof. This is immediate from (3.6), the definitions of $I_{1}$ and $a_{K}(A)$, and the following fact on modules over a Dedekind domain: if $\mathfrak{a}_{1}, \ldots, \mathfrak{a}_{t}$ are nonzero ideals in a Dedekind domain $R$, then the direct sum $\mathfrak{a}_{1} \oplus \cdots \oplus \mathfrak{a}_{t}$ is $R$-free if and only if the ideal product $\mathfrak{a}_{1} \ldots \mathfrak{a}_{t} \subset R$ is a principal ideal [18].

(5.6) Proposition. The following three assertions are equivalent:

(a) the field $l\left(I_{1}\right)^{\pi}$ is rational over $l^{\pi}$;

(b) the field $l\left(I_{1}\right)^{\pi}$ is stably rational over $l^{\pi}$;

(c) condition (i) of the main theorem is satisfied.

Proof. From the definitions of $I_{1}$ and (3.3) it is clear that (2.6) may be applied to $M=I_{1}$. Therefore it suffices to prove that condition (c) of (2.6), with $M=I_{1}$, is equivalent to condition (i) of the main theorem. But this is precisely (5.5).

Proof of the Main Theorem. First suppose $k_{A}$ is rational over $k$. Then $l(I)^{\pi}$ is stably rational over $k$, by (5.3). Using (5.4) and (1.8) we find $I \oplus N_{1} \cong N_{2}$ for some permutation modules $N_{1}$ and $N_{2}$ over $\pi$. From (1.1) and (3.2) we conclude that $n(q)=0$ if $\rho(q)$ is non-cyclic, that is, we have proved (ii) of the main theorem. It follows that $I=I_{1}$, and applying (5.6) we find that (i) is also satisfied.

Secondly, assume that (i) and (ii) of the main theorem hold. Then $I=I_{1}$ and $(5.6)$ teils us that $l(I)^{n}$ is rational over $i^{\pi}=k$. Application of $(5.3)$ concludes the proof.

(5.7) Remark. Note that the proof implies: if $k_{A}$ is stably rational over $k$, then $k_{A}$ is rational over $k$, for abelian $A$.

\section{Supplementary Results}

Two extension fields $K$ and $L$ of a field $k$ are called stably isomorphic over $k$ if there exist rational field extensions $K \subset K^{t}$ and $L \subset L^{\prime}$ of finite transcendence degree, such that $K^{\prime}$ and $L^{\prime}$ are $k$-isomorphic.

Let $k$ be a field, and $A$ and $A^{\prime}$ finite abelian groups. Write

$$
A^{\prime} \cong P^{\prime} \oplus B^{\prime}, \quad B^{\prime} \cong \underset{q}{\oplus}(\mathbf{Z} / q \mathbf{Z})^{n^{\prime}(\boldsymbol{q})}
$$

just as we did for $A$ in Sections 4 and 5 . 
(6.1) Theorem. Let $k$ be a field, and $A$ and $A^{\prime}$ finite abelian groups. Then $k_{A}$ and $k_{A^{\prime}}$ are stably isomorphic over $k$ if and only if the following two conditions are satisfied:

(i) for every intermediate field $k \subset K \subset k_{\text {cycl }}$ for which (0.1) holds, the $\mathbf{Z}\left(\rho_{K}\right)$-ideals $\mathfrak{a}_{K}(A)$ and $\mathfrak{a}_{K}\left(A^{\prime}\right)$ are in the same ideal class;

(ii) if $\operatorname{char}(k) \neq 2$, then $n(q)=n^{\prime}(q)$ for every power of two $q=2^{s}$ for which the Galois group of $k\left(\zeta_{q}\right)$ over $k$ is non-cyclic.

Proof. Analogous to the proof of the main theorem.

Following Burnside, we consider a generalization of the problem posed in the introduction. Let $k$ be a field, $A$ a finite group, and $V$ a finitely generated faithful $k[A]$-module. The symmetric algebra of $V$ over $k$ is denoted by $S_{k}(V)$. The field of fractions $k(V)$ of $S_{k}(V)$ is rational over $k$ of transcendence degree $\operatorname{dim}_{k}(V)$, and the $A$-action on $V$ induces an action of $\boldsymbol{A}$ on $k(\boldsymbol{V})$ as a group of field automorphisms over $k$. We ask under which conditions $k(V)^{A}$ is rational over $k$. If $V$ has a $k$-basis which is permuted by $A$, this is the question of the introduction. For $A$ abelian and $V=k[A]$, as $k[A]$-module, the answer is given by the main theorem. Theorem (6.4) below gives a partial solution for abelian $A$.

(6.2) Proposition. Let $V$ be a finitely generated faithful $k[A]$-module, and $W \subset V$ a faithful $k[A]$-submodule. Then $k(V)^{A}$ is rational over $k(W)^{A}$.

Proof. This follows easily from (1.3). Compare [33].

(6.3) Proposition. Suppose $A \cong P \oplus B$, where $|\boldsymbol{P}|$ is a power of char $(k)$ and $|B| \neq 0$ mod char $(k)$. Let $V$ be a finitely generated faithful $k[A]$ module. Then $V^{P}$ is a faithful $k[B]$-module, and $k(V)^{A}$ is rational over $k\left(V^{P}\right)^{\boldsymbol{B}}$.

Proof. We show that $V^{P}$ is a faithful $k[B]$-module. Let $b \in B$, with $b \neq 1$. Then $(b-1) V$ is a nonzero $P$-module, so by $(4.3)$ there is a nonzero element $w \in(b-1) V \cap V^{P}$, say $w=(b-1) v$. Let $m$ be the order of $b$. Then $b \cdot w=w$ would imply $m \cdot w=\left(b^{m-1}+\cdots+b+1\right) w=\left(b^{m}-1\right) v=0$, but $m \cdot 1 \neq 0$ in $k$, so $w=0$, contradiction. Hence $b \cdot w \neq w$, and $V^{P}$ is faithful over $k[B]$. The proof that $k(V)^{A}$ is rational over $k\left(V^{P}\right)^{B}$ follows exactly the same lines as the proof of (4.1).

(6.4) Theorem. Let $k$ be a field, A a finite abelian group, and $V$ a finitely generated faithful $k[A]$-module. Then $k(V)^{A}$ is stably rational over $k$ if and only if $k_{A}$ is rational over $k$. Moreover, if $\operatorname{dim}_{k}(V) \geqq|A|$, then $k(V)^{A}$ is rational over $k$ if and only if $k(V)^{A}$ is stably rational over $k$.

Proof. Write $A=P \oplus B$ as in (6.3). Combination of (6.3) and (6.2) (with $W=V^{P}$ ) shows that $k(V)^{A}$ and $k(V)^{B}$ are $k$-isomorphic, so it suffices to do the case $A=B$, i.e. $|A| \neq 0 \bmod \operatorname{char}(k)$. 
By (6.2), the field $k(V \oplus k[A])^{A}$ is rational over both $k\left(V^{4}\right)^{A}$ and $k_{A}$, so $k(V)^{A}$ and $k_{A}$ are stably isomorphic over $k$. Also, by (5.7), the field $k_{A}$ is rational over $k$ if and only if it is stably rational over $k$. We conclude that $k(V)^{A}$ is stably rational over $k$ if and only if $k_{A}$ is rational over $k$.

Finally, assume $\operatorname{dim}_{k}(V) \geqq|A|$. We may write $V \cong \bigoplus_{i=1} V_{i}^{n(i)}$ over $k[A]$, where each $V_{i}$ is an irreducible $k[A]$-module, $n(i)$ is a positive integer, and $V_{i}$ and $V_{j}$ are non-isomorphic for $i \neq j$. Put $W=\overbrace{i=1}^{t} V_{i}$. Then $W$ is a faithful $k[A]$-module, and there are injective $k[A]$-homomorphisms $W \rightarrow V$ and $W \rightarrow k[A]$. Therefore $k_{A}$ and $k(V)^{A}$ are both rational over $k(W)^{A}$. Since $\operatorname{dim}_{k}(V) \geqq|A|$, it follows that $k(V)^{A}$ is $k$-isomorphic to a rational extension of $k_{A}$. Application of (5.7) completes the proof. $]$

The argument in our solution of the case $V=k[A]$ which does not carry over to the general case is the proof of (5.2). But by exercising a little more care one can show that the bound $|A|$ in (6.4) may be replaced by $|A|-|\Phi(A)|$, where $\Phi(A)$ denotes the Frattini subgroup of $A$ (i.e., the intersection of the maximal subgroups of $A$ ).

\section{Corollaries}

We note some consequences of our main theorem. Some of them appeared already in $[10,48]$.

(7.1) Corollary. Let $k$ be a field and $p$ a prime number. The splitting field of $X^{p}-1$ over $k$ is denoted by $l$, and $d=[l: k]$. Then $k_{\mathbf{z}_{p} \mathbf{z}}$ is rational over $k$ if and only if the ring $\mathbf{Z}\left[\zeta_{d}\right]$ contains a principal ideal of index $p$.

Proof. We may assume $2 \neq p \neq \operatorname{char}(k)$. By the main theorem, $k_{\mathbf{z} / p \mathbf{z}}$ is rational over $k$ if and only if $\mathfrak{a}_{l}(\mathbf{Z} / p \mathbf{Z})$ is a principal ideal of $\mathbf{Z}\left(\rho_{l}\right)$. This implies (7.1), since $a_{l}(\mathbf{Z} / p \mathbf{Z})$ has index $p$ in $\mathbf{Z}\left(\rho_{l}\right) \cong \mathbf{Z}\left[\zeta_{d}\right]$ and since any two ideals of index $p$ in $\mathbf{Z}\left[\zeta_{d}\right]$ are conjugate over $\mathbf{Z}$.

(7.2) Corollary. Let $n \geqq 1$ be an integer. Then $\mathbf{Q}_{\mathbf{z}_{i n \mathbf{z}}}$ is rational over $\mathbf{Q}$ if and only if the following two conditions are satisfied:

(i) the integer $n$ is not divisible by 8 ;

(ii) for every divisor $q$ of $n$ of the form $q=p^{s}$, with $p$ an odd prime and $s$ a positive integer, the ring $\mathbf{Z}\left[\zeta_{\phi(q)}\right]$ contains a principal ideal of index $p$; here $\phi(q)=p^{s-1} \cdot(p-1)$.

Proof. This is just a translation of the main theorem for this case. $]$

(7.3) Corollary. Let $k$ be a field and A a finite abelian group such that the exponent of $A$ divides

$2^{2} \cdot 3^{m} \cdot 5^{2} \cdot 7^{2} \cdot 11 \cdot 13 \cdot 17 \cdot 19 \cdot 23 \cdot 29 \cdot 31 \cdot 37 \cdot 41 \cdot 43 \cdot 61 \cdot 67 \cdot 71$

for some non-negative integer $m$. Then $k_{A}$ is rational over $k$. 
Proof. It suffices to show that for each odd prime power $q=p^{s}$ dividing the exponent of $A$ the ring $\mathbf{Z}\left[\zeta_{\phi(q)}\right]$ contains an element of norm $p$. This has been done in $[10]$

(7.4) Corollary. Let $k$ be a field and $A$ a finite abelian group such that:

(i) for every odd prime $p$ which divides the exponent of $A$, the splitting field of $X^{p}-1$ over $k$ has degree 1 or 2 over $k$;

(ii) if $r$ is the highest power of 2 dividing the exponent of $A$, then the splitting field of $X^{r}-1$ over $k$ is a cyclic extension of $k$.

Then $k_{A}$ is rational over $k$.

Proof. This follows from the main theorem and the remark that $1-\zeta_{p^{t}}$ is an element of norm $p$ in the ring $\mathbf{Z}\left[\zeta_{p^{t}}\right]=\mathbf{Z}\left[\zeta_{2 p^{t}}\right]$, for every odd prime power $p^{t}$.

Corollary (7.4) confirms a conjecture of Kuniyoshi [32] for $p \neq 2$; for $p=2$ the conjecture is false.

(7.5) Corollary. Let $k$ be $a$ field and $A$ a finite abelian group. Assume that condition (ii) of the main theorem is satisfied. Then there exists a rational field extension $k \subset L$ of finite transcendence degree, and a Galois extension $L \subset L^{\prime}$, such that $\mathrm{Gal}\left(L^{\prime} / L\right) \cong A$.

Proof. Let $e$ be the exponent of $A$, and let $l$ be the splitting field of $X^{e}-1$ over $k$. Denote by $h$ the lowest common multiple of the class numbers of the rings $\mathbf{Z}\left(\rho_{K}\right)$, where $K$ runs over the fields $k \subset K \subset l$ which are cyclic over $k$. Put $G=A^{h}$. Then the main theorem implies that $k_{G}$ is rational over $k$. Hence we can take $L=k_{G}$ and $L$ equal to a suitable intermediate field $k_{G} \subset L^{\prime} \subset k\left(\left\{x_{g} \mid g \in G\right\}\right)$.

(7.6) Corollary. Let $k$ be a field, which, as a field, is finitely generated over its prime field. Let $P_{k}$ denote the set of prime numbers $p$ for which $k_{\mathbf{z} / p \mathbf{z}}$ is rational over $k$. Then $P_{k}$ has Dirichlet density 0 inside the set of all prime numbers.

Proof (sketch). We need some algebraic number theory [26].

First we consider the case $\operatorname{char}(k)=0$. Then $\left[k\left(\zeta_{p}\right): k\right]=p-1$ for all but finitely many prime numbers $p$, so by (7.1) it suffices to do the case $k=\mathbf{Q}$.

For a prime number $m$, let $K_{m}=\mathbf{Q}\left(\zeta_{m}\right)$, let $L_{m}$ be the Hilbert class field of $K_{m}$, and let $h(m)=\left[L_{m}: K_{m}\right]$ be the class number of $K_{m}$. We put

$S_{m}=\left\{p \mid p\right.$ is a prime number, which either splits completely in $L_{m}$, or does not split completely in $\left.K_{m}\right\} \cup\{m\}$.

We claim $P_{\mathbf{Q}} \subset S_{m}$, for every prime number $m$. In fact, if $p \in P_{\mathbf{Q}}$ is a prime number unequal to $m$ which splits completely in $K_{m}$, then $m \mid p-1$; but 
by (7.1) the ring $\mathbf{Z}\left[\zeta_{p-1}\right]$ contains a principal ideal of norm $p$, and therefore also $\mathbf{Z}\left[\zeta_{m}\right]$ contains a principal ideal of norm $p$. This means that $p$ splits completely in $L_{m}$, as required.

Using Tchebotarev's theorem and an easily proved linear disjointness statement, we find that for any finite set $M$ of prime numbers the set $\cap S_{m}$ has Dirichlet density $m \in M$

$$
\prod_{m \in M}(1-(h(m)-1) / h(m) \cdot(m-1)) .
$$

Since $h(m) \geqq 2$ for all prime numbers $m \geqq 23$, cf. [29], it follows that $\bigcap_{m \text { prime }} S_{m}$ has Dirichlet density 0 . Therefore also the subset $P_{\mathbf{Q}}$ of $\bigcap_{m \text { prime }} S_{m}$ has Dirichlet density 0.

The case of nonzero characteristic is slightly more complicated. We may assume that $k$ is a finite field, say $k=\mathbf{F}_{q}$, where $q=r^{n}$ and $r=\operatorname{char}(k)$.

For a prime number $m$, let $K_{m}, L_{m}$ and $h(m)$ be as above, and put $E_{m}=K_{m}\left(q^{1 / m}\right)$. We define:

$$
\begin{aligned}
& T_{m}=\left\{p \mid p \text { is a prime number, which splits completely in } L_{m},\right. \\
& \\
& \quad \text { or splits completely in } E_{m}, \\
&\text { or does not split completely in } \left.K_{m}\right\} \cup\{m, r\} .
\end{aligned}
$$

We show $P_{k} \subset T_{m}$ for every prime number $m$. Namely, assume that $p \in P_{k}$ does not divide $m r$ and splits completely in $K_{m}$. We distinguish two cases. If the order of $(q \bmod p) \in \mathbf{F}_{p}^{*}$ is divisible by $m$, then $\left[k\left(\zeta_{p}\right): k\right]$ is divisible by $m$. Using (7.1), we then conclude in the same way as for $k=\mathbf{Q}$ that $p$ splits completely in $L_{m}$, so $p \in T_{m}$. On the other hand, if the order of $(q \bmod p)$ in $\mathbf{F}_{p}^{*}$ is not divisible by $m$, then it is relatively prime to $m$, so $(q \bmod p)$ is an $m$-th power in $\mathbf{F}_{p}$. Since we assumed that $p$ splits completely in $K_{m}$, this implies that $p$ splits completely in $E_{m}$, so $p \in T_{m}$, as required.

If $M$ is any finite set of prime numbers $m$ not dividing $n \cdot r$, the Dirichlet density of $\bigcap_{m \in M} T_{m}$ is

Hence

$$
\prod_{m \in M}(1-(h(m)-1) / h(m) \cdot m) .
$$

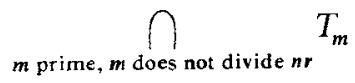

has Dirichlet density 0 , so the same is true for $P_{k}$. $\quad$,

Finally, we remark that for $k=\mathbf{F}_{2}$ the set $P_{k}$ contains all Mersenne and Fermat prime numbers.

Acknowledgements. The author is greatly indebted to Prof. F. Oort and Prof. W. Kuyk, without whose stimulating help this paper never would have been written. 


\section{References}

1. Artin, E.: The Orders of the Linear Groups. Comm. Pure Appl. Math. 8, 355-366 (1955)

2. Bourbaki, N.: Algèbre, Ch. IV et V. Paris: Hermann 1967

3. Breuer, S.: Zyklische Gleichungen 6. Grades und Minimalbasis Math. Ann. 86, $108-113(1922)$

4. Breuer, S.: Zur Bestimmung der metazyklischen Minimalbasis von Primzahlgrad. Math. Ann. 92, 126-144 (1924)

5. Breuer, S.: Metazyklische Minimalbasis und komplexe Primzahlen. 3. Reine Angew. Math. 156, 13-42 (1927)

6. Cassels. J.W.S., Fröhlich, A. (eds.): Algebraic Number Theory. London-New York: Academic Press 1967

7. Charnow, A.: On the fixed field of a linear abelian group. J. London Math. Soc. (2) 1 , 348-350 (1969)

8. Chevalley, C.: Invariants of finite groups generated by reflections. Amer. J, Math. 77, 778-782 (1955)

9. Demazure, M.: Sous-groupes algébriques de rang maximum du groupe de Cremona Ann. Sci. École Norm. Sup. (4) 3, 507-588 (1970)

10. Endo, S., Miyata, T.: Invariants of finite abelian groups. J. Math. Soc. Japan 25, 7-26 (1973)

11. Endo, S., Miyata, T.: Quasi-permutation modules over finite groups. J. Math. Soc. Japan 25, 397-421 (1973)

12. Fischer, E.: Die Isomorphie der Invariantenkörper der endlichen Abel'schen Gruppen linearer Transformationen. Nachr. Königl. Ges. Wiss. Göttingen, 77-80 (1915)

13. Fischer, E.: Zur Theorie der endlichen Abelschen Gruppen. Math. Ann. 77,81-88 (1916)

14. Furtwängler, P.: Über Minimalbasen für Körper rationaler Funktionen. S.-B. Akad. Wiss. Wien 134, 69-80 (1925)

15. Galkin, V.M.: On an ideal in the group ring of a cyclic group (Russian). Uspehi Mat. Nauk 28, 233-234 (1973)

16. Gaschütz, W.: Fixkörper von p-Automorphismengruppen rein-transzendenter Körpererweiterungen von $p$-Charakteristik. Math. Z. 71, 466-468 (1959)

17. Haeuslein, G.: On the invariants of finite groups having an abelian normal subgroup of prime index. J. London Math. Soc. (2) 3, 355-360 (1971)

18. Kaplansky, I.: Modules over Dedekind rings and valuation rings. Trans. Amer. Math Soc. $72,327-340$ (1952)

19. Kervaire, M.: Fractions rationnelles invariantes. Séminaire Bourbaki, exp. 445 (1973/1974)

20. Kuniyoshi, H.: On purely-transcendency of a certain field. Tôhoku Math. J. 6, 101-108 (1954)

21. Kuniyoshi, H.: On a problem of Chevalley. Nagoya Math. J. 8, 65-67 (1955)

22. Kuniyoshi, H.: Certain subfields of rational function fields. In: Proc. int. symp. algebraic number theory, pp. 241-243. Tokyo-Nikko: 1955

23. Kuyk, W.: Over het omkeerprobleem van de Galoistheorie. Thesis, Amsterdam 1960

24. Kuyk, W.: On a theorem of E. Noether. Nederl. Akad. Wetensch. Proc. Ser. A 67, 32-39 (1964)

25. Kuyk, W.. Mullender, P.: On the invariants of finite abelian groups. Nederl. Akad. Wetensch. Proc. Ser. A 66, 232-237 (1963)

26. Lang, S.: Algebraic Number Theory. Reading: Addison-Wesley 1970

27. Lenstra, Jr., H.W.: Rational functions invariant under a finite abelian group. Report 72-02, Dept. of Math., University of Amsterdam (1972)

28. Martinet, J.: Un contre-exemple à une conjecture d'E. Noether (d'après R. Swan). Seminaire Bourbaki, exp. 372 (1969/1970), In: Lecture Notes in Mathematics 180, pp. 145-154. Berlin-Heidelberg-New York: Springer 1971 
29. Masley, J.M.: On the class number of cyclotomic fields. Thesis, Princeton University 1972

30. Masuda, K.: On a problem of Chevalley. Nagoya Math. J. 8, 59-63 (1955)

31. Masuda, K.: Application of the theory of the group of classes of projective modules to the existence problem of independent parameters of invariant. J. Math. Soc. Japan 20, 223-232(1968)

32. Matsuda, R.: On purely-transcendency of certain fields. Tôhoku Math. J. 16, 189-202 (1964)

33. Miyata, T.: Invariants of certain groups, I. Nagoya Math. J. 41, 69-73 (1971)

34. Nagata, M.: A theorem on valuation rings and its applications. Nagoya Math. J.29, 85-91 (1967)

35. Noether, E.: Rationale Funktionenkörper. Jber. Deutsch. Math.-Verein. 22, 316-319 (1913)

36. Noether, E.: Körper und Systeme rationaler Funktionen. Math. Ann. 76, 161-196 (1915)

37. Noether, E.: Gleichungen mit vorgeschriebener Gruppe. Math. Ann. 78, 221-229(1918)

38. Ono, T.: Arithmetic of algebraic tori. Ann. of Math. 74, 101-139 (1961)

39. Ono, T.: On the Tamagawa number of algebraic tori. Ann. of Math. 78, 47 73 (1963)

40. Segre, B.: Sur un problème de M. Zariski. In: Colloque d'Algèbre et de Théorie des Nombres, pp. 135-138. Paris: C.N.R.S. 1950

41. Seidelmann, F.: Die Gesamtheit der kubischen und biquadratischen Gleichungen mit Affekt bei beliebigem Rationalitätsbereich. Thesis, Erlangen 1916. Summary: Math. Ann. 78, 230-233 (1918)

42. Serre, J,-P.: Corps Locaux. Paris: Hermann 1962

43. Speiser, A.: Zahientheoretische Sätze aus der Gruppentheorie. Math. Z. 5, 1-6 (1919)

44. Swan, R. G.: Invariant Rational Functions and a Problem of Steenrod. Invent. Math. 7, 148-158 (1969)

45. Voskresenskii, V.E.: Birational properties of linear algebraic groups (Russian). Izv. Akad. Nauk SSSR Ser. Mat. 34, 3-19 (1970). English translation: Math. USSR - Izv. 4, $1-17(1970)$

46. Voskresenskii, V.E.: On the question of the structure of the subfield of invariants of a cyclic group of automorphisms of the field $\mathbf{Q}\left(x_{1}, \ldots, x_{n}\right)$ (Russian). Izv. Akad. Nauk SSSR Ser. Mat. 34, 366-375 (1970). English translation: Math. USSR - Izv. 4, 371-380 (1970)

47. Voskresenskii, V.E.: Rationality of certain algebraic tori (Russian). Izv. Akad. Nauk SSSR Ser. Mat. 35, 1037-1046 (1971). English translation: Math. USSR - Izv. 5, 1049-1056 (1971)

48. Voskresenskií, V.E.: Fields of invariants of abelian groups (Russian). Uspehi Mat. Nauk 28, 77-102(1973)

H.W. Lenstra, Jr.

Mathematisch Instituut

Roetersstraat 15

Amsterdam

The Netherlands

(Received February 27, 1974) 